\title{
ASYMPTOTIC GEOMETRY OF NEGATIVELY CURVED MANIFOLDS OF FINITE VOLUME
}

\author{
F. Dal'Bo, M. Peigné, J.C. Picaud \& A. Sambusetti
}

20, January 2018

\begin{abstract}
We study the asymptotic behaviour of simply connected, Riemannian manifolds $X$ of strictly negative curvature admitting a non-uniform lattice $\Gamma$. If the quotient manifold $\bar{X}=\Gamma \backslash X$ is asymptotically $1 / 4$-pinched, we prove that $\Gamma$ is divergent and $U \bar{X}$ has finite Bowen-Margulis measure (which is then ergodic and totally conservative with respect to the geodesic flow); moreover, we show that, in this case, the volume growth of balls $B(x, R)$ in $X$ is asymptotically equivalent to a purely exponential function $c(x) e^{\delta R}$, where $\delta$ is the topological entropy of the geodesic flow of $\bar{X}$. This generalizes Margulis' celebrated theorem to negatively curved spaces of finite volume. In contrast, we exhibit examples of lattices $\Gamma$ in negatively curved spaces $X$ (not asymptotically $1 / 4$-pinched) where, depending on the critical exponent of the parabolic subgroups and on the finiteness of the Bowen-Margulis measure, the growth function is exponential, lower-exponential or even upper-exponential.
\end{abstract}

AMS classification : 53C20, 37C 35

Keywords: Cartan-Hadamard manifold, volume, entropy, Bowen-Margulis measure

\section{Introduction}

Let $X$ be a complete, simply connected manifold with strictly negative curvature. In the sixties, G. Margulis [25], using measure theory on the foliations of the Anosov system defined by the geodesic flow, showed that if $\Gamma$ is a uniform lattice of $X$ (i.e. a torsionless, discrete group of isometries such that $\bar{X}=\Gamma \backslash X$ is compact), then the orbital function of $\Gamma$ is asymptotically equivalent ${ }^{1}$ to a purely exponential function:

$$
v_{\Gamma}(x, y, R)=\#\{\gamma \in \Gamma \mid d(x, \gamma y)<R\} \sim c_{\Gamma}(x, y) e^{\delta(\Gamma) R}
$$

where $\delta(\Gamma)=\lim _{R \rightarrow \infty} R^{-1} \ln v_{\gamma}(x, x, R)$ is the critical exponent of $\Gamma$. By integration over fundamental domains, one then obtains an asymptotic equivalence for the volume growth function of $X$ :

$$
v_{X}(x, R)=\operatorname{vol} B(x, R) \sim m(x) e^{\delta(\Gamma) R} .
$$

\footnotetext{
${ }^{1}$ Given two functions $f, g: \mathbb{R}_{+} \rightarrow \mathbb{R}_{+}$, we say that $f$ is asymptotically equivalent to $g$ when $\lim _{R \rightarrow+\infty} f(R) / g(R)=1$, and we will write $f \sim g$.
} 
It is well-known that the exponent $\delta(\Gamma)$ equals the topological entropy of the geodesic flow of $\bar{X}$ (see [27]) and that, for uniform lattices, it is the same as the volume entropy $\omega(X)=\lim \sup \frac{1}{R} \ln v_{X}(x, R)$ of the manifold $X$. The function $m(x)$, depending on the center of the ball, is the Margulis function of $X$.

Since then, this result has been generalized in different directions. Notably, G. Knieper showed in [24] that the volume growth function of a Hadamard space $X$ (a complete, simply connected manifolds with nonpositive curvature) of rank one admitting uniform lattices is purely exponential ${ }^{2}$, that is

$$
v_{X}(x, R) \asymp e^{\omega(X) R}
$$

In general, he showed that $v_{X}(x, R) \asymp R^{\frac{d-1}{2}} e^{\omega(X) R}$ for rank $d$ manifolds; however, as far as the authors are aware, it is still unknown whether there exists a Margulis function for Hadamard manifolds of rank 1 with uniform lattices, i.e. a function $m(x)$ such that $v_{X}(x, R) \sim m(x) e^{\omega(X) R}$, even in the case of surfaces. Another remarkable case is that of asymptotically harmonic manifolds of strictly negative curvature, where the strong asymptotic homogeneity implies the existence of a Margulis function, even without compact quotients, cp. [11].

In another direction, it seems natural to ask what happens for a Hadamard space $X$ of negative curvature admitting nonuniform lattices $\Gamma$ (i.e. $\operatorname{vol}(\Gamma \backslash X)<\infty$ ): is $v_{X}$ purely exponential and, more precisely, does $X$ admit a Margulis function? Let us emphasize that if $X$ also admits a uniform lattice then $X$ is a symmetric space of rank one (by [18], Corollary 9.2.2); therefore, we are interested in spaces which do not have uniform lattices, i.e. the universal covering of finite volume, negatively curved manifolds which are not locally symmetric.

It is worth to stress here that the orbital function of $\Gamma$ is closely related to the volume growth function of $X$, but it generally has, even for lattices, a different asymptotic behaviour than $v_{X}(x, R)$. A precise asymptotic equivalence fo $v_{\Gamma}$ was proved by $\mathrm{T}$. Roblin [30] in a very general setting, proving a dichotomy based on the finiteness of the so-called Bowen-Margulis measure associated to $\Gamma$.

In order to state this dichotomy, let us recall some general definitions. The limit set of a general non elementary discrete subgroup $\Gamma$ of isometries of $X$ is the subset $\Lambda(\Gamma) \subset$

\footnotetext{
${ }^{2}$ We will systematically use the following convenient notation in the paper: given two functions $f, g: \mathbb{R}_{+} \rightarrow \mathbb{R}_{+}$, we write $f \stackrel{C}{\prec} g$ for $R>R_{0}$ (or $g \stackrel{C}{\succ} f$ ) if there exists $C>0$ such that $f(R) \leq C g(R)$ for these values of $R$. Similarly, $f \asymp C$ means $g \stackrel{C}{\prec} f \stackrel{C}{\prec} g$. We simply write $f \prec g$ and $f \asymp g$ when the constants $C$ and $R_{0}$ are unessential.

The upper and lower exponential growth rates of the function $f$ are respectively defined as:$$
\omega^{+}(f)=\limsup _{R \rightarrow+\infty} R^{-1} \ln f(R) \quad \text { and } \quad \omega^{-}(f)=\omega(f)=\liminf _{R \rightarrow+\infty} R^{-1} \ln f(R)
$$

and we simply write $\omega(f)$ when the two limits coincide.

Finally, we say that $f$ is purely exponential if $f \asymp e^{\omega(f) R}$, lower-exponential when $\limsup _{R \rightarrow+\infty} \frac{f(R)}{e^{\omega(f) R}}=0$ and upper-exponential when $\liminf _{R \rightarrow+\infty} \frac{f(R)}{e^{\omega(f) R}}=+\infty$; when the weaker property $\limsup _{R \rightarrow+\infty} \frac{f(R)}{e^{\omega(f) R}}=+\infty$ holds, we say that $f$ is weakly upper-exponential.
} 
$X(\infty)$ of accumulation points of any orbit $\Gamma \cdot x$ in $X$. By Patterson's construction (see [30] for a precise description), there exists on $\Lambda(\Gamma)$ a family $\left(\mu_{x}\right)_{x \in X}$ of finite measures, supported by $\Lambda(\Gamma)$, satisfying the following conditions: for any $x, x^{\prime} \in X$ and any $g \in \Gamma$,

$$
\frac{\mathrm{d} \mu_{\mathrm{x}}}{\mathrm{d} \mu_{\mathrm{x}^{\prime}}}(\xi)=e^{-\delta(\Gamma) b_{\xi}\left(x, x^{\prime}\right)} \quad \text { and } \quad \mu_{\gamma^{-1} x}=\gamma_{*} \mu_{x}
$$

where $b_{\xi}\left(x, x^{\prime}\right)=\lim _{y \rightarrow \xi} d(x, y)-d\left(x^{\prime}, y\right)$ is the Busemann function centered at $\xi \in \Lambda(\Gamma)$.

When identifying the unit tangent bundle of $X$ with $(X(\infty) \times X(\infty)-\Delta) \times \mathbb{R}$ (where $\Delta$ denotes the diagonal in $X(\infty) \times X(\infty)$ ), these two properties readily imply that the measure $e^{-\delta(\Gamma)\left(b_{\eta}(x, y)+b_{\xi}(x, y)\right)} d \eta d \xi d t$ is a Radon measure on $U X$, which is invariant under the actions of both $\Gamma$ and the translation flow on the third coordinate; thus, it induces a measure $\mu_{B M}$ on the unit tangent bundle $U \bar{X}$ of the quotient manifold $\bar{X}=\Gamma \backslash X$, which is invariant for the geodesic flow and called the Bowen-Margulis measure.

T. Roblin proved that for any non-elementary discrete group of isometries $\Gamma$ of a CAT(-1) space $X$ with non-arithmetic length spectrum ${ }^{3}$, one has:

(a) $v_{\Gamma}(x, y, R) \sim c_{\Gamma}(x, y) e^{\delta(\Gamma) R}$ if the measure $\mu_{B M}$ is finite;

(b) $v_{\Gamma}(x, y, R)=o(R) e^{\delta(\Gamma) R}$, where $o(R)$ is infinitesimal, otherwise.

Thus, the behaviour of $v_{\Gamma}(x, R)$ strongly depends on the finiteness of the measure $\mu_{B M}$; also, the asymptotic constant can be expressed in terms of $\mu_{B M}$ and of the family of Patterson-Sullivan measures $\left(\mu_{x}\right)$ of $\Gamma$, as $c_{\Gamma}(x, y)=\frac{\left\|\mu_{x}\right\|\left\|\mu_{y}\right\|}{\delta(\Gamma) \cdot\left\|\mu_{B M}\right\|}$.

In this paper we restrict our attention to lattices $\Gamma$, which are fundamental examples of geometrically finite groups; let us describe this class. Let $C(\Gamma)$ be the convex hull in $X$ of the limit set $\Lambda(\Gamma)$ in $X \cup X(\infty)$; the group $\Gamma$ acts properly discontinuously on $C(\Gamma)$, the quotient $\bar{N}(\Gamma)=\Gamma \backslash C(\Gamma)$ is called the Nielsen core of $\bar{X}$. The group $\Gamma$ (or the quotient manifold $\bar{X}$ ) is said to be geometrically finite when for some $\varepsilon>0$ the $\varepsilon$-neighborhood $\bar{N}_{\varepsilon}(\Gamma)$ of $\bar{N}(\Gamma)$ has finite volume. We refer to [8] for a complete description of geometrical finiteness in variable negative curvature. When $\Gamma$ is a lattice, the Nielsen core $\bar{N}(\Gamma)$ equals $\bar{X}$, thus $\Gamma$ is clearly geometrically finite. In contrast, the groups considered in [29] are generally not geometrically finite. In section $\S 4$ we will recall a useful criterion (Finiteness Criterion (15), due to Dal'BoOtal-Peigné), to decide whether a geometrically finite group has $\mu_{B M}(U \bar{X})<\infty$ or not; hence, a precise asymptotics for $v_{\Gamma}(x, R)$ as in (a).

On the other hand, any convergent group $\Gamma$ exhibits a behaviour as in (b), since it certainly has infinite Bowen-Margulis measure (by Poincaré recurrence, $\mu_{B M}(U \bar{X})<\infty$ implies that the geodesic flow is totally conservative, and this is equivalent to divergence, by Hopf-Tsuji-Sullivan's theorem). Notice that, whereas uniform lattices always are divergent and with finite Bowen-Margulis measure, for nonuniform lattices $\Gamma$ divergence and condition (15) in general may fail. Namely, this can happen only in case $\Gamma$ has a "very large" parabolic subgroup $P$, that is such that $\delta(P)=\delta(\Gamma)$ : we will call exotic such a lattice $\Gamma$, and we will say that such a $P$ is a dominant parabolic subgroup.

\footnotetext{
${ }^{3}$ This means that the additive subgroup of $\mathbb{R}$ generated by the length of closed geodesics in $G \backslash X$ is dense in $\mathbb{R}$; it is the case, for instance, if $\operatorname{dim}(X)=2$, or when $G=\Gamma$ is a lattice.
} 
Convergent, exotic lattices are constructed by the authors in [16]; also, one can find in [16] some original counting results for the orbital function of $\Gamma$ in infinite BowenMargulis measure, more precise than (b).

However, as we shall see, the volume growth function $v_{X}$ has a wilder behaviour than $v_{\Gamma}$. In [14] we proved that for nonuniform lattices in pinched, negatively curved spaces $X$, the functions $v_{\Gamma}$ and $v_{X}$ can have different exponential growth rates, i.e. $\omega(X) \neq \delta(\Gamma)$. In the Example 5.2 we will see that the function $v_{X}$ might as well have different superior and inferior exponential growth rates $\omega^{ \pm}(X)$ (notice, in contrast, that $\delta(\Gamma)$ always is a true limit).

The main result of the paper concerns finiteness of the Bowen-Margulis measure and an aymptote for the volume growth function of $\frac{1}{4}$-pinched spaces with lattices:

Theorem 1.1 Let $X$ be a Hadamard space with curvature $-b^{2} \leq K_{X} \leq-a^{2}$, and let $\Gamma$ be a nonuniform lattice of $X$. If $\bar{X}=\Gamma \backslash X$ has asymptotically $1 / 4$-pinched curvature (that is, for any $\epsilon>0$, the metric satisfies $-k_{+}^{2} \leq K_{X} \leq-k_{-}^{2}$ with $k_{+}^{2} \leq 4 k_{-}^{2}+\epsilon$ outside some compact set $\left.\bar{C}_{\epsilon} \subset \bar{X}\right)$, then:

(i) $\Gamma$ is divergent and the Bowen-Margulis measure $\mu_{B M}$ of $U \bar{X}$ is finite;

(ii) $\omega^{+}(X)=\omega^{-}(X)=\delta(\Gamma)$;

(iii) there exists a function $\bar{m}(x) \in L^{1}(\bar{X})$ such that $v_{X}(x, R) \sim m(x) e^{\delta(\Gamma) R}$, where $m(x)$ is the lift of $\bar{m}$ to $X$.

From (i) it follows that the geodesic flow of any asymptotically $\frac{1}{4}$-pinched, negatively curved manifold of finite volume is ergodic and totally conservative w.r. to $\mu_{B M}$, by Hopf-Tsuji-Sullivan Theorem (see [33], [30]), contrary to the case of general negatively curved manifolds of finite volume (e.g., those obtained from convergent lattices). By [27], it also follows that $\mu_{B M}$ is the unique measure of maximal entropy for the geodesic flow on $U \bar{X}$ in this case.

Condition (iii) also implies that volume equidistributes on large spheres, i.e. the volume $v_{X}^{\Delta}(x, R)$ of annuli in $X$ of thickness $\Delta$ satisfies the precise asymptotic law:

$$
v_{X}^{\Delta}(x, R) \sim 2 m(x) \sinh (\Delta \delta(\Gamma)) e^{\omega(X) R}
$$

The above theorem also covers the classical case of noncompact symmetric spaces of rank one (where the proof of the divergence and the asymptotics is direct).

One may wonder about the meaning of the $\frac{1}{4}$-pinching condition. This turns out to be an asymptotic, geometrical condition on the influence and wildness of parabolic subgroups of $\Gamma$ associated to the cusps of $\bar{X}=\Gamma \backslash X$. Parabolic groups, being elementary, do not necessarily have a critical exponent which can be interpreted as a true limit; rather, for a parabolic group of isometries $P$ of $X$, one can consider the limits

$$
\delta^{+}(P)=\limsup _{R \rightarrow \infty} \frac{1}{R} \ln v_{P}(x, R), \quad \delta^{-}(P)=\liminf _{R \rightarrow \infty} \frac{1}{R} \ln v_{P}(x, R)
$$

and the critical exponent $\delta(P)$ of the Poincaré series of $P$ coincides with $\delta^{+}(P)$. The parabolic group $P$ is called maximal when it is not a proper subgroup of some 
parabolic subgroup of $\Gamma$. Accordingly, we say that a lattice $\Gamma$ is sparse if it has a maximal parabolic subgroup $P$ such that $\delta^{+}(P)>2 \delta^{-}(P)$ (conversely, we will say that $\Gamma$ is parabolically $\frac{1}{2}$-pinched if it is not sparse). Such parabolic groups in $\Gamma$, together with dominant parabolic subgroups, are precisely associated to cusps whose growth can wildly change, and this can globally influence the growth function of $X$. Namely, in section 4 , we prove :

Theorem 1.2 Let $X$ be a Hadamard manifold with pinched, negative curvature $-b^{2} \leq K_{X} \leq-a^{2}<0$. If $X$ has a nonuniform lattice $\Gamma$ which is neither exotic nor sparse, then $\Gamma$ is divergent with finite Bowen-Margulis measure; moreover, $v_{X} \asymp v_{\Gamma}$ and $X$ has a Margulis function $m(x)$, whose projection is $L^{1}$ on $\bar{X}=\Gamma \backslash X$.

The divergence and finiteness of the Bowen-Margulis measure in Theorem 1.1 and Theorem 1.2 are both consequence of a critical gap between $\delta(\Gamma)$ and the exponential growth rates $\delta^{+}\left(P_{i}\right)$ of all parabolic subgroups; this will be proved in $\S 4$. In particular, we will see that any lattice $\Gamma$ in a negatively curved, $\frac{1}{4}$-pinched space is never exotic (nor sparse). For this, we will use an asymptotic characterization of the hyperbolic lattices as the only lattices in spaces $X$ with pinched curvature $-b^{2} \leq K_{X} \leq-a^{2}$ realizing the least possible value for the topological entropy of $\bar{X}=\Gamma \backslash X$, i.e. satisfying $\delta(\Gamma)=(n-1) a$. In the compact case, this result can be deduced from Knieper's work on spherical means (following the proof of Theorem $5.2,[24]$ ), or from Bonk-Kleiner [4] (for convex-cocompact groups); on the other hand, see [17] for a complete proof in the case of non-uniform lattices and the analysis of the new difficulties arising in the non-compact case.

The existence of the Margulis function in Theorems 1.1 and 1.2 relies on a Counting Formula (Proposition 3.1), proved in $\S 3$; the formula enables us to reduce the computation of $v_{X}$ to the analytic profile of the cusps of $\bar{X}$ and $v_{\Gamma}$ (so, in the last instance, to T.Roblin's asymptotics $(a) \mathcal{E}(b))$.

The last part of the paper is devoted to studying sparse and exotic lattices, to understand the necessity of the $\frac{1}{4}$-pinching (or $\frac{1}{2}$-parabolically pinching) conditions. The following result shows that Theorem 1.2 is the best that we can expect for Hadamard spaces with quotients of finite volume.

Theorem 1.3 Let $X$ be a Hadamard manifold with pinched negative curvature $-b^{2} \leq K_{X} \leq-a^{2}<0$ admitting a nonuniform lattice $\Gamma$.

(i) If $\Gamma$ is exotic and the dominant subgroups $P$ satisfy $\delta(\Gamma)=\delta^{+}(P)<2 \delta^{-}(P)$, then both $v_{X}$ and $v_{\Gamma}$ are purely exponential or lower-exponential, with the same exponential growth rate $\omega(X)=\delta(\Gamma)$. Namely:

- either $\mu_{B M}=\infty$, and in this case $v_{X}$ is lower-exponential.

- or $\mu_{B M}<\infty$, and then $v_{X}$ is purely exponential and $X$ has a Margulis function;

The two cases can actually occur, cp. Examples 5.3(a)\&(b).

(ii) If $\Gamma$ is exotic and a dominant subgroup $P$ satisfies $\delta(\Gamma)=\delta^{+}(P)=2 \delta^{-}(P)$, then $\omega(X)=\delta(\Gamma)$ but in general $v_{X} \nprec v_{\Gamma}$, and $X$ does not admit a Margulis function. Namely, there exist cases (Examples 5.4(a)E(b)) where: 
- $\mu_{B M}<\infty$, with $v_{\Gamma}$ purely exponential and $v_{X}$ upper-exponential;

- $\mu_{B M}=\infty$, with $v_{\Gamma}$ lower-exponential and $v_{X}$ upper-exponential.

Notice that, in all cases under consideration in this theorem, the equality $\omega(X)=\delta(\Gamma)$ holds, by Theorem 1.2 in [14], since $\delta^{+}(P) \leq 2 \delta^{-}(P)$ (cp. also Corollary 3.3). The proof of assertion (i) of Theorem 1.3 is given in section $\S 4$, with explicit examples in section $\S 5$ (Examples 5.3); the examples proving assertion (ii) are also developed in section $\S 5$ (Examples 5.4). We shall also see that all the examples mentioned in this statement can be obtained as lattices in $\left(\frac{1}{4}-\epsilon\right)$-pinched spaces, for arbitrary $\epsilon>0$, which shows the optimality of the $\frac{1}{4}$-pinching condition.

On the other hand, if $\Gamma$ is sparse, one can even have $\omega^{+}(X)>\omega^{-}(X)>\delta(\Gamma)$, and the Example 5.2 shows that virtually any asymptotic behaviour for $v_{X}$ can occur. Thus, the case of exotic lattices with a parabolic subgroup such that $\delta^{+}(P)=2 \delta^{-}(P)$ can be seen as the critical threshold where a transition happens, from functions $v_{\Gamma}, v_{X}$ with same asymptotic behaviour to functions with even different exponential growth rate.

Notice at last that the condition $\delta^{+}(P)<2 \delta^{-}(P)$ is satisfied when $\frac{b^{2}}{a^{2}}<\frac{1}{4}$, and that this last condition implies that the group $P$ is abelian [4].

\section{Growth of parabolic subgroups and of lattices modulo parabolic subgroups}

Throughout all the paper, unless otherwise stated, $X$ will be a Hadamard space of dimension $n$, with pinched negative sectional curvature $-b^{2} \leq K_{X} \leq-a^{2}<0$.

For $x, y \in X$ and $\xi$ belonging to the geometric boundary $X(\infty)$, we will denote $[x, y]$ (resp. $[x, \xi]$ ) the geodesic segment from $x$ to $y$ (resp. the ray from $x$ to $\xi$ ). We will repeatedly make use of the following, classical result in strictly negative curvature (see for instance [9]): there exists $\epsilon(a, \vartheta)=\frac{1}{|a|} \log \left(\frac{2}{1-\cos \vartheta}\right)$ such that any geodesic triangle $x y z$ in $X$ making angle $\vartheta=\angle_{z}(x, y)$ at $z$ satisfies:

$$
d(x, y) \geq d(x, z)+d(z, x)-\epsilon(a, \vartheta) .
$$

Let $b_{\xi}(x, y)=\lim _{z \rightarrow \xi} d(x, z)-d(z, y)$ be the Busemann function centered at $\xi$. The level set $\partial H_{\xi}(x)=\left\{y \mid b_{\xi}(x, y)=0\right\}$ (resp. the suplevel set $H_{\xi}(x)=\left\{y \mid b_{\xi}(x, y) \geq 0\right\}$ is the horosphere (resp. the horoball) with center $\xi$ and passing through $x$. From (1) we easily deduce the following:

Lemma 2.1 For any $d>0$, there exists $\epsilon_{1}=\epsilon_{1}(a, d) \geq \epsilon\left(a, \frac{\pi}{2}\right)$ with the following property: given two disjoint horoballs $H_{1}, H_{2}$ at distance $d=d\left(H_{1}, H_{2}\right)=d\left(z_{1}, z_{2}\right)$ with $z_{i} \in \partial H_{i}$, then for any $x \in H_{1}$ and $y \in H_{2}$ we have

$$
d\left(x, z_{1}\right)+d\left(z_{1}, z_{2}\right)+d\left(z_{2}, y\right)-\epsilon_{1}(a, d) \leq d(x, y) \leq d\left(x, z_{1}\right)+d\left(z_{1}, z_{2}\right)+d\left(z_{2}, y\right) .
$$

Proof. As $K_{X} \leq-a^{2}$ and horoballs are convex, for any $y \in H_{2}$ the angle $\vartheta(y)=\angle_{z_{1}} z_{2}, y$ satisfies $\tan \vartheta(y) \leq \frac{1}{\sinh (d /|a|)}$ (cp. for instance [31], Prop.8). Then, 
we have $\angle_{z_{1}} x, y \geq \frac{\pi}{2}-\vartheta(y) \geq \vartheta(d)$ with $\vartheta(d)>0$ for $d \neq 0$, hence, by (1),

$$
d(x, y) \geq d\left(x, z_{1}\right)+d\left(z_{1} y\right)-\epsilon(a, \vartheta(d)) \geq d\left(x, z_{1}\right)+d\left(z_{1}, z_{2}\right)+d\left(z_{2}, y\right)-\epsilon_{1}(a, d)
$$

for $\epsilon_{1}(a, d)=\epsilon(a, \vartheta(d))+\epsilon\left(a, \frac{\pi}{2}\right)$.

Let $d_{\xi}$ denote the horospherical distance between two points on a same horosphere centered at $\xi$. If $\psi_{\xi, t}: X \rightarrow X$ denotes the radial flow in the direction of $\xi$, we define:

$$
t_{\xi}(x, y)= \begin{cases}\inf \left\{t>0 \mid d_{\xi}\left(\psi_{\xi, t+\Delta}(x), \psi_{\xi, t}(y)\right)<1\right\} & \text { if } b_{\xi}(x, y)=\Delta \geq 0 \\ \inf \left\{t>0 \mid d_{\xi}\left(\psi_{\xi, t}(x), \psi_{\xi, t-\Delta}(y)\right)<1\right\} & \text { if } b_{\xi}(x, y)=\Delta<0\end{cases}
$$

If $y$ is closer to $\xi$ than $x$, let $x_{\Delta}=\left[x, \xi\left[\cap \partial H_{\xi}(y)\right.\right.$ : then, $t_{\xi}(x, y)$ represents the minimal time we need to apply the radial flow $\psi_{\xi, t}$ to the points $x_{\Delta}$ and $y$ until they are at horospherical distance less than 1 . Using (1) and the lower curvature bound $K_{X} \geq-b^{2}$, we obtain in [14] the following estimate, which is also crucial in our computations:

\section{Approximation Lemma $\mathbf{2 . 2}$}

There exists $\epsilon_{0}=\epsilon_{0}(a, b) \geq \epsilon\left(a, \frac{\pi}{2}\right)$ such that for all $x, y \in X$ and $\xi \in X(\infty)$ we have:

$$
2 t_{\xi}(x, y)+\left|b_{\xi}(x, y)\right|-\epsilon_{0} \leq d(x, y) \leq 2 t_{\xi}(x, y)+\left|b_{\xi}(x, y)\right|+\epsilon_{0}
$$

In this section we give estimates for the growth of annuli in a parabolic subgroup and in quotients of a lattice by a parabolic subgroup, which will be used later. So, let us fix some notations. We let $A^{\Delta}(x, R)=B\left(x, R+\frac{\Delta}{2}\right) \backslash B\left(x, R-\frac{\Delta}{2}\right)$ be the annulus of radius $R$ and thickness $\Delta$ around $x$. For a group $\Gamma$ of isometries of $X$, we will consider the orbital functions

$$
v_{G}(x, y, R)=\#(B(x, R) \cap G y) \quad v_{G}^{\Delta}(x, y, R)=\#\left(A^{\Delta}(x, R) \cap G y\right)
$$

and we set $v_{G}(x, R)=v_{G}(x, x, R), v_{G}^{\Delta}(x, R)=v_{G}^{\Delta}(x, x, R)$ and $v_{G}^{\Delta}(x, R)=\emptyset$ for $\Delta<0$. We will also need to consider the growth function of coset spaces, endowed with the natural quotient metric: if $H<G$, we define $d_{x}\left(g_{1} H, g_{2} H\right):=d\left(g_{1} H x, g_{2} H x\right)$ and

$$
\begin{gathered}
v_{G / H}(x, R):=\#\left\{\left.g H|| g H\right|_{x}=d_{x}(H, g H)<R\right\} \\
v_{G / H}^{\Delta}(x, R)=v_{G / H}\left(x, R+\frac{\Delta}{2}\right)-v_{G / H}\left(x, R-\frac{\Delta}{2}\right) .
\end{gathered}
$$

We will use analogous notations for the growth functions of balls and annuli in the spaces of left and double cosets $H \backslash G, H \backslash G / H$ with the metrics

$$
\begin{gathered}
d_{x}\left(H g_{1}, H g_{2}\right):=d\left(H g_{1} x, H g_{2} x\right)=\left|g_{1}^{-1} H g_{2}\right|_{x} \\
d_{x}\left(H g_{1} H, H g_{2} H\right):=d\left(H g_{1} H x, H g_{2} H x\right)=\left|g_{1}^{-1} H g_{2} H\right|_{x} .
\end{gathered}
$$

The growth of the orbital function of a bounded parabolic group $P$ is best expressed by introducing the horospherical area function. Let us recall the necessary definitions: 
Definitions 2.3 Let $P$ be a bounded parabolic group of $X$ fixing $\xi \in X(\infty)$ : that is, acting cocompactly on $X(\infty)-\{\xi\}$ (as well as on every horosphere $\partial H$ centered at $\xi$ ). Given $x \in X$, let $\mathcal{D}(P, x)$ be a Dirichlet domain centered at $x$ for the action of $P$ on $X$; that is, a convex fundamental domain contained in the closed subset

$$
\overline{\mathcal{D}}(P, x)=\{y \in X \mid d(x, y) \leq d(p x, y) \text { for all } p \in P\}
$$

We set $\mathcal{S}_{x}=\mathcal{D}(P, x) \cap \partial H_{\xi}(x)$ and $\mathcal{C}_{x}=\mathcal{D}(P, x) \cap H_{\xi}(x)$, and denote by $\mathcal{S}_{x}(\infty)$ the trace at infinity ${ }^{4}$ of $\mathcal{D}(P, x)$, minus $\xi$; these are, respectively, fundamental domains for the actions of $P$ on $\partial H_{\xi}(x), H(x)$ and $X(\infty)-\{\xi\}$.

The horospherical area function of $P$ is the function

$$
\mathcal{A}_{P}(x, R)=\operatorname{vol}\left[P \backslash \psi_{\xi, R}\left(\partial H_{\xi}(x)\right)\right]=\operatorname{vol}\left[\psi_{\xi, R}\left(\mathcal{S}_{x}\right)\right]
$$

where the vol is the Riemannian measure of horospheres. We also define the cuspidal function of $P$, which is the function

$$
\mathcal{F}_{P}(x, R)=\operatorname{vol}\left[B(x, R) \cap H_{\xi}(x)\right]
$$

that is, the volume of the intersection of a ball centered at $x$ and the horoball centered at $\xi$ and passing through $x$. Notice that the functions $\mathcal{A}_{P}(x, R), \mathcal{F}_{P}(x, R)$ only depend on the choice of the initial horosphere $\partial H_{\xi}(x)$.

REMARK 2.4 Well-known estimates of the differential of the radial flow (cp. [21]) yield, when $-b^{2} \leq K_{X} \leq-a^{2}<0$,

$$
e^{-b t}\|v\| \leq\left\|d \psi_{\xi, t}(v)\right\| \leq e^{-a t}\|v\|
$$

Therefore we deduce that, for any $\Delta>0$,

$$
e^{-(n-1) b \Delta} \leq \frac{\mathcal{A}_{P}(x, R+\Delta)}{\mathcal{A}_{P}(x, R)} \leq e^{-(n-1) a \Delta}
$$

The following Propositions show how the horospherical area $\mathcal{A}_{P}$ and the cuspidal function $\mathcal{F}_{P}$ are related to the orbital function of $P$; they refine and precise some estimates given in [14] for $v_{P}(x, R)$.

Proposition 2.5 Let $P$ be a bounded parabolic group of $X$ fixing $\xi$, with $\operatorname{diam}\left(\mathcal{S}_{x}\right) \leq d$. There exist $C=C(n, a, b, d)$ and $C^{\prime}=C^{\prime}(n, a, b, d ; \Delta)$ such that:

$$
\begin{array}{lll}
v_{P}(x, y, R) \stackrel{C}{\smile} \mathcal{A}_{P}^{-1}\left(x, \frac{R+b_{\xi}(x, y)}{2}\right) & \forall R \geq b_{\xi}(x, y)+R_{0} \\
v_{P}^{\Delta}(x, y, R) \stackrel{C^{\prime}}{\smile} \mathcal{A}_{P}^{-1}\left(x, \frac{R+b_{\xi}(x, y)}{2}\right) & \forall R \geq b_{\xi}(x, y)+R_{0} \text { and } \forall \Delta>\Delta_{0}
\end{array}
$$

for explicit constants $R_{0}$ and $\Delta_{0}$ only depending on $n, a, b, d$.

\footnotetext{
${ }^{4}$ The trace at infinity $A(\infty)$ of a subset $A \subset X$ is defined as the intersection of $X(\infty)$ with the closure of $A$ in $X \cup X(\infty)$.
} 
Proposition 2.6 Same assumptions as in Proposition 2.5. We have:

$$
\mathcal{F}_{P}(x, R) \subseteq \int_{0}^{R} \frac{\mathcal{A}_{P}(x, t)}{\mathcal{A}_{P}\left(x, \frac{R+t}{2}\right)} d t \quad \forall R \geq R_{0}
$$

REMARK 2.7 More precisely, we will prove (and use later) that:

(i) $v_{P}(x, y, R) \stackrel{C}{\prec} \mathcal{A}_{P}^{-1}\left(x, \frac{R+b_{\xi}(x, y)}{2}\right)$ for all $R>0$;

(ii) $v_{P}^{\Delta}(x, y, R) \stackrel{C^{\prime}}{\prec} \mathcal{A}_{P}^{-1}\left(x, \frac{R+b_{\xi}(x, y)}{2}\right)$ for all $\Delta, R>0$;

(iv) $\mathcal{F}_{P}(x, R) \stackrel{C}{\prec} \int_{0}^{R} \frac{\mathcal{A}_{P}(x, t)}{\mathcal{A}_{P}\left(x, \frac{R+t}{2}\right)} d t$ for all $R>0$.

As a direct consequence of (7) and (5) we have (see also Corollary 3.5 in [14]):

Corollary 2.8 Let $P$ be a bounded parabolic group of $X$. Then:

$$
\delta^{-}(P) \leq \omega^{-}\left(\mathcal{F}_{P}\right) \leq \omega^{+}\left(\mathcal{F}_{P}\right) \leq \max \left\{\delta^{+}(P), 2\left(\delta^{+}(P)-\delta^{-}(P)\right)\right\}
$$

Proof of Proposition 2.5. Since $v_{P}(x, y, R)=v_{P}(y, x, R)$ and $\mathcal{A}_{P}(x, R)=$ $\mathcal{A}_{P}\left(y, R-b_{\xi}(x, y)\right)$, we can assume that $t=b_{\xi}(x, y) \geq 0$. If $z \in \partial H_{\xi}(y)$ and $d(x, z)=R$, we know by Lemma 2.2 that $2 t_{\xi}(x, z)+t-\epsilon_{0} \leq d(x, z) \leq 2 t_{\xi}(x, z)+t+\epsilon_{0}$, so $\left|t_{\xi}(x, z)-\frac{R-t}{2}\right| \leq \epsilon_{0} / 2$. We deduce that $d_{\xi}\left(\psi_{\xi, \frac{R+t+\epsilon_{0}}{2}}(x), \psi_{\xi, \frac{R-t+\epsilon_{0}}{2}}(z)\right) \leq 1$, so the set $\psi_{\xi, \frac{R-t+\epsilon_{0}}{2}}\left(B(x, R) \cap \partial H_{\xi}(y)\right)$ is contained in the unitary ball $B^{+}$of the horosphere $\partial H_{\xi}\left(x^{+}\right)$, centered at $x^{+}=\psi_{\xi, \frac{R+t+\epsilon_{0}}{2}}(x)$. Similarly, if $R>t+\epsilon_{0}$ then $t_{\xi}(x, z)>0$, so $d_{\xi}\left(\psi_{\xi, \frac{R+t-\epsilon_{0}}{2}}(x), \psi_{\xi, \frac{R-t-\epsilon_{0}}{2}}(z)\right) \geq 1$, and the set $\psi_{\xi, \frac{R-t-\epsilon_{0}}{2}}\left(B(x, R) \cap \partial H_{\xi}(y)\right)$ contains the unitary ball $B^{-}$of $\partial H_{\xi}\left(x^{-}\right)$, centered at the point $x^{-}=\psi_{\xi, \frac{R+t-\epsilon_{0}}{2}}(x)$. We know that, by Gauss' equation, the sectional curvature of horospheres of $X^{2}$ is between $a^{2}-b^{2}$ and $2 b(b-a)$ (see, for instance, [5], §1.4); therefore, there exist positive constants $v^{-}=v^{-}(a, b)$ and $v^{+}=v^{+}(a, b)$ such that $\operatorname{vol}\left(B^{+}\right)<v^{+}$and $\operatorname{vol}\left(B^{-}\right)>v^{-}$. Now, let $\mathcal{S}_{y}=\psi_{\xi, t}\left(\mathcal{S}_{x}\right)$ be the fundamental domain for the action of $P$ on $\partial H_{\xi}(y)$ deduced from $\mathcal{S}_{x}$. There are at least $v_{\mathcal{P}}(x, y, R-d)$ distinct fundamental domains $p \mathcal{S}_{y}$ included in $B(x, R) \cap \partial H_{\xi}(y)$; since the radial flow $\psi_{\xi, t}$ is equivariant with respect to the action of $P$ on the horospheres centered at $\xi$, there are also at least $v_{P}(x, y, R-d)$ distinct fundamental domains $\psi_{\xi, \frac{R-t+\epsilon_{0}}{2}}\left(p \mathcal{S}_{y}\right)$ included in $\psi_{\xi, \frac{R-t+\epsilon_{0}}{2}}\left(B(x, R) \cap \partial H_{\xi}(y)\right)$. We deduce that $v_{P}(x, y, R-d) \cdot \mathcal{A}_{P}\left(x, \frac{R+t+\epsilon_{0}}{2}\right)<v^{+}$and, by $(4)$, this gives $v_{P}(x, y, R) \stackrel{C}{\prec}$ $\mathcal{A}_{P}^{-1}\left(x, \frac{R+t}{2}\right)$ for all $R \geq 0$. On the other hand, if $R>t+\epsilon_{0}$, we can cover the set $B(x, R) \cap \partial H_{\xi}(y)$ with $v_{P}(x, y, R+d)$ fundamental domains $p \mathcal{S}_{y}$, with $p \in P$; then, again, $\psi_{\xi, \frac{R-t-\epsilon_{0}}{2}}\left(B(x, R) \cap \partial H_{\xi}(y)\right)$ can be covered by $v_{P}(x, y, R+d)$ fundamental domains $\psi_{\xi, \frac{R-t-\epsilon_{0}}{2}}\left(p \mathcal{S}_{y}\right)$ as well, hence we deduce that $v_{P}(x, y, R+d) \cdot \mathcal{A}_{P}\left(x, \frac{R+t-\epsilon_{0}}{2}\right) \geq v^{-}$. This implies that $v_{P}(x, y, R) \stackrel{C}{\succ} \mathcal{A}_{P}^{-1}\left(x, \frac{R+t}{2}\right)$ for all $R>t+R_{0}$, for $R_{0}=\epsilon_{0}+d$ and a constant $C=C(n, a, b, d)$. 
To prove the weak equivalence (6), we just write, for $R+\frac{\Delta}{2}>t+R_{0}$ :

$$
\begin{aligned}
v_{P}^{\Delta}(x, y, R) & =v_{P}^{\Delta}(x, y, R+\Delta / 2)-v_{P}^{\Delta}(x, y, R-\Delta / 2) \geq \frac{C^{-1}}{\mathcal{A}_{P}\left(\frac{R+t+\Delta / 2}{2}\right)}-\frac{C}{\mathcal{A}_{P}\left(\frac{R+t-\Delta / 2}{2}\right)} \\
& \geq \frac{C^{-1} e^{(n-1) a \frac{\Delta}{4}}-C e^{-(n-1) a \frac{\Delta}{4}}}{\mathcal{A}_{P}\left(x, \frac{R+t}{2}\right)}=2 \sinh \left[\frac{1}{4}(n-1) a \Delta-\ln C\right] \cdot \mathcal{A}_{P}^{-1}\left(\frac{R+t}{2}\right)
\end{aligned}
$$

again by (4), if $\Delta>\Delta_{0}=\frac{4 \ln C}{(n-1) a}$. Reciprocally, we have for all $R, \Delta>0$ :

$$
v_{P}^{\Delta}(x, y, R) \leq v_{P}\left(x, y, R+\frac{\Delta}{2}\right) \leq \frac{C}{\mathcal{A}_{P}\left(x, \frac{R+t+\Delta / 2}{2}\right)} \leq \frac{C^{\prime}(n, a, b, d ; \Delta)}{\mathcal{A}_{P}\left(x, \frac{R+t}{2}\right)}
$$

Proof of Proposition 2.6. We just integrate (5) over a fundamental domain $\mathcal{C}_{x}$ for the action of $P$ on $H_{\xi}(x)$ :

$$
\mathcal{F}_{P}(x, R)=\sum_{p \in P} \operatorname{vol}\left[B(x, R) \cap p \mathcal{C}_{x}\right]=\int_{\mathcal{C}_{x}} \sum_{p \in P} 1_{B(x, R)}(p z) d z=\int_{\mathcal{C}_{x}} v_{P}(x, y, R) d y
$$

so, integrating over each slice $\psi_{\xi, t}\left(\mathcal{S}_{x}\right)$ by the coarea formula, we obtain

$$
\int_{0}^{R-R_{0}} \int_{\psi_{\xi, t}\left(\mathcal{S}_{x}\right)} \mathcal{A}_{P}^{-1}\left(x, \frac{R+t}{2}\right) d t \stackrel{C}{\prec} \mathcal{F}_{P}(x, R) \stackrel{C}{\prec} \int_{0}^{R} \int_{\psi_{\xi, t}\left(\mathcal{S}_{x}\right)} \mathcal{A}_{P}^{-1}\left(x, \frac{R+t}{2}\right) d t
$$

(the left inequality holding for $R>R_{0}$ ). By (4), both sides are $\asymp$ to the integral $\int_{0}^{R} \frac{\mathcal{A}_{P}(x, t)}{\mathcal{A}_{P}\left(x, \frac{R+t}{2}\right)} d t$, up to a multiplicative constant $c=c(n, a, b, d)$.

REMARK 2.9 Thus, we see that the curvature bounds imply that $v_{P}^{\Delta}(x, R) \asymp v_{P}(x, R)$ for $\Delta$ and $R$ large enough. This also holds in general for non-elementary groups $\Gamma$ with finite Bowen-Margulis measure, as in this case $v_{\Gamma}^{\Delta}(x, R) \sim \frac{2\left\|\mu_{x}\right\|^{2}}{\left\|\mu_{B M}\right\|} \sinh \left[\frac{\Delta}{2} \delta(\Gamma)\right] e^{\delta(\Gamma) R}$ by Roblin's asymptotics. On the other hand, it is unclear whether the weak equivalence $v_{\Gamma}^{\Delta} \asymp v_{\Gamma}$ holds for non-elementary lattices $\Gamma$, when $\left\|\mu_{B M}\right\|=\infty$.

In the next section we will also need estimates for the growth of annuli in the spaces of left and right cosets of a lattice $\Gamma$ of $X$, modulo a bounded parabolic subgroup $P$. Notice that, if $P$ fixes $\xi \in X(\infty)$, the function $v_{P \backslash \Gamma}(x, R)$ counts the number of points $\gamma x \in \Gamma x$ falling in the Dirichlet domain $\mathcal{D}(P, x)$ of $P$ with $d(x, \gamma x)<R$; on the other hand, the function $v_{\Gamma / P}(x, R)$ counts the number of horoballs $\gamma H_{\xi}(x)$ at distance (almost) less than $R$ from $x$. It is remarkable that, even if these functions count geometrically distinct objects, they are weakly asymptotically equivalent, as the following Proposition will show. Actually, let $H_{\xi}$ be a horoball centered at the parabolic fixed point $\xi$ of $P<\Gamma$; we call $\operatorname{depth}\left(H_{\xi}\right)$ the minimal distance $\min _{\Gamma-P} d\left(H_{\xi}, \gamma H_{\xi}\right)$. Then, for $\mathcal{S}_{x}$ defined as in Definition 2.3 we have:

Proposition 2.10 Let $\Gamma$ be a torsionless, non-elementary, discrete group of isometries of $X$, let $P$ a bounded parabolic subgroup of $\Gamma$, and let $x \in X$ be fixed. Assume that 
$\max \left\{\operatorname{diam}\left(\mathcal{S}_{x}\right), 1 / \operatorname{depth}\left(H_{\xi}(x)\right)\right\} \leq d$, and let $\ell$ be the minimal displacement $d(x, \gamma x)$ of the elements $\gamma \in \Gamma$ whose domains of attraction $\mathcal{U}^{ \pm}(\gamma, x)=\left\{y \mid d\left(\gamma^{ \pm 1} x, y\right) \leq d(x, y)\right\}$ are included in the Dirichlet domain $\mathcal{D}(P, x)$.

Then, there exists a constant $\delta_{0}=\delta_{0}(a, d)$ such that, for all $\Delta, R>0$ :

(i) $v_{P \backslash \Gamma}^{\Delta-\delta_{0}}(x, R) \leq v_{\Gamma / P}^{\Delta}(x, R) \leq v_{P \backslash \Gamma}^{\Delta+\delta_{0}}(x, R)$;

(ii) $\frac{1}{2} v_{\Gamma}^{\Delta-2 \ell}(x, R) \leq v_{P \backslash \Gamma}^{\Delta}(x, R) \leq v_{\Gamma}^{\Delta}(x, R)$;

(iii) $\frac{1}{2} v_{\Gamma}^{\Delta-\delta_{0}-2 \ell}(x, R) \leq v_{\Gamma / P}^{\Delta}(x, R) \leq v_{\Gamma}^{\Delta+\delta_{0}}(x, R)$;

(iv) $\frac{1}{4} v_{\Gamma}^{\Delta-\delta_{0}-4 \ell}(x, R) \leq v_{P \backslash \Gamma / P}^{\Delta}(x, R) \leq v_{\Gamma}^{\Delta}(x, R)$.

Notice that (iv) strenghtens a result of S. Hersonsky and F. Paulin on the number of rational lines with depth smaller than $R$ (cp. [20] Theorem 1.2, where the authors furthermore assume the condition $\delta_{P}<\delta_{\Gamma}$ ). Actually, let $H_{\xi}$ be the largest horosphere centered at $\xi$ non intersecting any other $\gamma H_{\xi}$ for $\gamma \neq e$, and recall that the depth of a geodesic $c=(\xi, \gamma \xi)$ is defined as the length of the maximal subsegment $\hat{c} \subset c$ outside $\Gamma H_{\xi}$. The double coset space $P \backslash(\Gamma-P) / P$ can be identified with the set of oriented geodesics $(\xi, \gamma \xi)$ of $X$ with $\gamma \in \Gamma-P$. Then, if $x \in \partial H_{\xi}$, the counting function $v_{P \backslash(\Gamma-P) / P}^{\Delta}(x, R)$ corresponds to the number of geodesics of $\bar{X}=\Gamma \backslash X$ which travel a time about $R$ outside the cusp $\overline{\mathcal{C}}=P \backslash H_{\xi}$, before entering and definitely staying (in the future and in the past) in $\overline{\mathcal{C}}$.

Proof. The right-hand inequalities in (ii), (iii), (iv) are trivial.

Let us prove (i). We first define two sections of the projections $P \backslash \Gamma \leftarrow \Gamma \rightarrow \Gamma / P$. Consider the fundamental domain $\mathcal{S}_{x}(\infty)$ for the action of $P$ on $X(\infty)-\{\xi\}$ given in 2.3, and choose for each $\gamma \in \Gamma$, a representative $\hat{\gamma}$ of $\gamma P$ which minimizes the distance to $x$.

Then, we set

$$
\begin{gathered}
\widehat{\Gamma}=\{\widehat{\gamma} \mid \gamma P \in \Gamma / P\} \\
\Gamma_{0}=\left\{\gamma_{0} \mid \gamma_{0} \in \Gamma, \gamma_{0} \xi \in \mathcal{S}_{x}(\infty)\right\} \cup\{e\} .
\end{gathered}
$$

We have bijections $\widehat{\Gamma} \cong \Gamma / P$ and $\Gamma_{0} \cong P \backslash \Gamma$, as $\mathcal{S}_{x}(\infty)$ is a fundamental domain. Moreover, every $\gamma_{0} \in \Gamma_{0}$ almost minimizes the distance to $x$ in its right coset $P \gamma_{0}$. Actually, for all $\gamma \in \Gamma$ set $z(\gamma)=(\xi, \gamma \xi) \cap \partial H_{\xi}(x)$ and $z^{\prime}(\gamma)=(\xi, \gamma \xi) \cap \gamma \partial H_{\xi}(x)$; then, for all $p \in P$ we have, by Lemma 2.1

$d\left(x, p \gamma_{0} x\right) \geq d(x, p z(\gamma))+d\left(p z(\gamma), p z^{\prime}(\gamma)\right)+d\left(p z^{\prime}(\gamma), p \gamma_{0} x\right)-\epsilon_{1}(a, d) \geq d\left(x, \gamma_{0} x\right)-c$

as $d\left(H_{\xi}(x), p \gamma_{0} H_{\xi}(x)\right)=d\left(p z(\gamma), p z^{\prime}(\gamma)\right)$, for $c=2 d+\epsilon_{1}(a, d)$.

We will now define a bijection between pointed metric spaces $i:\left(P \backslash \Gamma, x_{0}\right) \rightarrow\left(\Gamma / P, x_{0}\right)$ which almost-preserves the distance to their base point $x_{0}=P$ (with respect to their quotient distances $|\cdot|_{x}=d_{x}(P, \cdot)$ as seen at the beginning of the section), as follows. For every $\gamma \in \Gamma$ we can write $\gamma=\widehat{\gamma} p_{\gamma}$, for uniquely determined $\widehat{\gamma} \in \widehat{\Gamma}$ and $p_{\gamma} \in P$; given a right coset $P \gamma$, we take $\gamma_{0} \in \Gamma_{0}$ representing $P \gamma$ and then set $i(P \gamma):=p_{\gamma_{0}} \widehat{\gamma}_{0} P$. The map $i$ is surjective. Actually, given $\gamma P$, we take $p \in P$ such that $p \gamma \xi \in \mathcal{S}_{x}(\infty)$, 
so that $P \gamma=P \gamma_{0}$, for $\gamma_{0}=p \gamma \in \Gamma_{0}$; then, we write $\gamma_{0}=\widehat{\gamma}_{0} p_{\gamma_{0}}$, and we deduce that $i(P \gamma)=i\left(P \gamma_{0}\right)=i\left(P \widehat{\gamma}_{0} p^{-1}\right)=p^{-1} \widehat{\gamma}_{0} P=p^{-1} \gamma_{0} p_{\gamma_{0}}^{-1} P=\gamma P$.

We now check that $i$ is injective. Given $\gamma_{0}=\widehat{\gamma}_{0} p_{\gamma_{0}}$ and $\gamma_{0}^{\prime}={\widehat{\gamma^{\prime}}}_{0} p_{\gamma_{0}^{\prime}}$ in $\Gamma_{0}$ representing two right cosets $P \gamma$ and $P \gamma^{\prime}$, assume that $p_{\gamma_{0}} \widehat{\gamma}_{0} P=p_{\gamma_{0}^{\prime}} \widehat{\gamma}_{0}^{\prime} P$. Then, $\widehat{\gamma}_{0} \xi=p \widehat{\gamma}_{0}^{\prime} \xi$ for $p=p_{\gamma_{0}}^{-1} p_{\gamma_{0}^{\prime}} \in P$, which yields $p_{\gamma_{0}}=p_{\gamma_{0}^{\prime}}$ as $\widehat{\gamma}_{0} \xi, \widehat{\gamma}^{\prime}{ }_{0} \xi \in \mathcal{S}_{x}(\infty)$ and $\mathcal{S}_{x}(\infty)$ is a fundamental domain for the left action of $P$; so, $\widehat{\gamma}_{0} P=\widehat{\gamma_{0}^{\prime}} P$, which implies that $\widehat{\gamma}_{0}=\widehat{\gamma_{0}^{\prime}}$ too $($ as $\widehat{\Gamma}$ is a section of $\Gamma / P)$. Therefore, $P \gamma=P \gamma_{0}=P \widehat{\gamma}_{0} p_{\gamma_{0}}=P{\widehat{\gamma^{\prime}}}_{0} p_{\gamma_{0}^{\prime}}=P \gamma_{0}^{\prime}=P \gamma^{\prime}$. To show that $i$ almost preserves ||$_{x}$, we notice that, given a class $P \gamma$ and writing its representative in $\Gamma_{0}$ as $\gamma_{0}=\widehat{\gamma}_{0} p_{\gamma_{0}}$, we have

$$
|P \gamma|_{x} \leq\left|\gamma_{0}\right|_{x} \leq d\left(x, \widehat{\gamma}_{0} x\right)+d\left(\widehat{\gamma}_{0} x, \widehat{\gamma}_{0} p_{\gamma_{0}} x\right)=\left|\widehat{\gamma}_{0}\right|_{x}+\left|p_{\gamma_{0}}\right|_{x}
$$

while, by (9) and by Lemma 2.1

$$
|P \gamma|_{x} \geq\left|\gamma_{0}\right|_{x}-c \geq d\left(x, z^{\prime}\left(\gamma_{0}\right)\right)+d\left(z^{\prime}\left(\gamma_{0}\right), \widehat{\gamma}_{0} p_{\gamma_{0}}\right)-\epsilon_{1}(a, d)-c \geq\left|\widehat{\gamma}_{0}\right|_{x}+\left|p_{\gamma_{0}}\right|_{x}-2 c
$$

as $d\left(z^{\prime}\left(\gamma_{0}\right), \widehat{\gamma}_{0} x\right)<d$. On the other hand

$$
|i(P \gamma)|_{x}=\left|p_{\gamma_{0}} \widehat{\gamma}_{0} P\right|_{x} \leq d\left(x, p_{\gamma_{0}} x\right)+d\left(p_{\gamma_{0}} x, p_{\gamma_{0}} \widehat{\gamma}_{0} P x\right)=\left|p_{\gamma_{0}}\right|_{x}+\left|\widehat{\gamma}_{0}\right|_{x}
$$

while, as $z\left(p_{\gamma_{0}} \widehat{\gamma}_{0}\right)=p_{\gamma_{0}} z\left(\widehat{\gamma}_{0}\right)$ and $z^{\prime}\left(p_{\gamma_{0}} \widehat{\gamma}_{0}\right)=p_{\gamma_{0}} z^{\prime}\left(\widehat{\gamma}_{0}\right)$, we get by Lemma 2.1

$$
|i(P \gamma)|_{x} \geq d\left(x, p_{\gamma_{0}} z\left(\widehat{\gamma}_{0}\right)\right)+d\left(p_{\gamma_{0}} z\left(\widehat{\gamma}_{0}\right), p_{\gamma_{0}} \widehat{\gamma}_{0} P x\right)-\epsilon_{1}(a, d) \geq\left|p_{\gamma_{0}}\right|_{x}+\left|\widehat{\gamma}_{0}\right|_{x}-c .
$$

This shows that $|P \gamma|_{x}-c \leq|i(P \gamma)|_{x} \leq|P \gamma|_{x}+2 c$. We then immediately deduce that $v_{P \backslash \Gamma}(x, R-2 c) \leq v_{\Gamma / P}(x, R) \leq v_{P \backslash \Gamma}(x, R+c)$, as well as (i) for $\delta_{0}=4 c$.

The proof of the left-hand inequality in (ii) is a variation for annuli of a trick due to Roblin, cp. [30]. Actually, as $L(P) \subsetneq L(\Gamma)$, we can choose a $\bar{\gamma} \in \Gamma$ with $d(x, \bar{\gamma} x)=\ell$ and such that the domains of attraction $\mathcal{U}^{ \pm}(\bar{\gamma}, x)$ are included in the domain $\mathcal{D}(P, x)$. Let $v_{\mathcal{D}(P, x)}(x, R)$ be the number of points of the orbit $\Gamma x$ falling in $\mathcal{D}(P, x) \cap B(x, R)$. We have:

$$
v_{\Gamma}^{\Delta}(x, R) \leq v_{\mathcal{D}(P, x)}^{\Delta}(x, R)+v_{\mathcal{D}(P, x)}^{\Delta+2 \ell}(x, R) \leq 2 v_{\mathcal{D}(P, x)}^{\Delta+2 \ell}(x, R)
$$

since, for $\gamma x \in A^{\Delta}(x, R)$, either $\gamma x \in \mathcal{D}(P, x)$, or $\bar{\gamma} \gamma x \in \mathcal{D}(P, x)$ and $\bar{\gamma} \gamma x \in A^{\Delta+2 \ell}(x, R)$. As the points of $P$ falling in $\mathcal{D}(P, x)$ minimize the distance to $x$ modulo the left action of $P$, we also have $v_{\mathcal{D}(P, x)}^{\Delta+2 \ell}(x, R)=v_{P \backslash \Gamma}^{\Delta+2 \ell}(x, R)$, which proves (ii).

Assertion (iii) follows directly from (i) and (ii). To show (iv), we need to estimate the number of classes $\gamma P$ modulo the left action of $P$, that is the elements of $\widehat{\Gamma}$ such that $\widehat{\gamma} x$ belongs to the fundamental domain $\mathcal{D}(P, x)$. We choose an element $\bar{\gamma} \in \Gamma$ with $\mathcal{U}^{ \pm}(\bar{\gamma}, x) \subset \mathcal{D}(P, x)$ as before, and apply again Roblin's trick to the classes $\gamma P$. The set $\widehat{\Gamma} x$ can be parted in two disjoint subsets: the subset $\widehat{\Gamma}_{1}:=\widehat{\Gamma} \cap \mathcal{D}(P, x)$, and the subset $\widehat{\Gamma}_{2}:=\widehat{\Gamma} \cap \mathcal{D}(P, x)^{c}$, whose elements $\widehat{\gamma}$ then satisfy $\bar{\gamma} \widehat{\gamma} \in \mathcal{D}(P, x)$ and $|\bar{\gamma} \widehat{\gamma}|_{x} \leq|\widehat{\gamma}|_{x}+\ell$. Then $v_{\Gamma / P}^{\Delta}(x, R)=v_{\widehat{\Gamma}_{1}}^{\Delta}(x, R)+v_{\widehat{\Gamma}_{2}}^{\Delta}(x, R) \leq 2 v_{P \backslash \Gamma / P}^{\Delta+2 \ell}(x, R)$ and we conclude by (iii). 


\section{Orbit-counting estimates for lattices}

In this section we give estimates of the orbital function $v_{\Gamma}(x, y, R)$ and of $v_{X}(R)$ in terms of the orbital function of the parabolic subgroups $P_{i}$ and the associated cuspidal functions $\mathcal{F}_{P_{i}}$ of $\Gamma$. These estimates will be used in $\S 4$ and $\S 5$; they stem from an accurate dissection of large balls in compact and horospherical parts, assuming that ambient space $X$ admits a nonuniform lattice action.

Let us introduce the following notation, which will be used throughout the next sections of the paper: if $f$ and $g$ are two real functions, the discrete convolution $f *_{\Delta} g$ of $f$ and $g$ with gauge $\Delta$ is defined by

$$
\left(f *_{\Delta} g\right)(R)=\sum_{h, k \geq 1}^{h+k=\lfloor R / \Delta\rfloor} f(h \Delta) g(k \Delta) .
$$

For nondecreasing functions $f$ and $g$, it clearly holds:

$$
\Delta \cdot\left(f *_{\Delta} g\right)(R-\Delta) \leq(f * g)(R)=\int_{0}^{R} f(t) g(R-t) d t \leq 2 \Delta \cdot\left(f *_{\Delta} g\right)(R+2 \Delta) .
$$

Let us recall some useful results due to B. Bowditch [8] concerning the structure of the limit set $L(\Gamma)$ and of $\bar{X}$ :

(a) $L(\Gamma)=X(\infty)$ and it is the disjoint union of the radial limit set $L_{r a d}(\Gamma)$ with finitely many orbits $L_{b p} \Gamma=\Gamma \xi_{1} \cup \ldots \cup \Gamma \xi_{m}$ of bounded parabolic fixed points; this means that each $\xi_{i} \in L_{b p} \Gamma$ is the fixed point of some maximal bounded parabolic subgroup $P_{i}$ of $\Gamma$;

(b) (Margulis' lemma) there exist closed horoballs $H_{\xi_{1}}, \ldots, H_{\xi_{m}}$ centered respectively at $\xi_{1}, \ldots, \xi_{m}$, such that $g H_{\xi_{i}} \cap H_{\xi_{j}}=\emptyset$ for all $1 \leq i, j \leq m$ and all $\gamma \in \Gamma-P_{i}$;

(c) $\bar{X}$ can be decomposed into a disjoint union of a compact set $\overline{\mathcal{K}}$ and finitely many "cusps" $\overline{\mathcal{C}}_{1}, \ldots, \overline{\mathcal{C}}_{m}$ : each $\overline{\mathcal{C}}_{i}$ is isometric to the quotient of $H_{\xi_{i}}$ by the maximal bounded parabolic group $P_{i} \subset \Gamma$. We refer to $\overline{\mathcal{K}}$ and to $\overline{\mathcal{C}}=\cup_{i} \overline{\mathcal{C}}_{i}$ as to the compact core and the cuspidal part of $\bar{X}$.

Throughout this section, we fix $x \in X$ and we consider a Dirichlet domain $\mathcal{D}(\Gamma, x)$ centered at $x$; this is a convex fundamental subset, and we may assume that $\mathcal{D}$ contains the geodesic rays $\left[x, \xi_{i}\left[\right.\right.$. Accordingly, setting $\mathcal{S}_{i}=\mathcal{D} \cap \partial H_{\xi_{i}}$ and $\mathcal{C}_{i}=\mathcal{D} \cap H_{\xi_{i}} \simeq \mathcal{S}_{i} \times \mathbb{R}_{+}$, the fundamental domain $\mathcal{D}$ can be decomposed into a disjoint union:

$$
\mathcal{D}=\mathcal{K} \cup \mathcal{C}_{1} \cup \cdots \cup \mathcal{C}_{m}
$$

where $\mathcal{K}$ is a convex, relatively compact set containing $x$ in its interior (projecting to a subset $\overline{\mathcal{K}}$ in $\bar{X}$ ), while $\mathcal{C}_{i}$ and $\mathcal{S}_{i}$ are, respectively, connected fundamental domains for the action of $P_{i}$ on $H_{\xi_{i}}$ and $\partial H_{\xi_{i}}$ (projecting respectively to subsets $\overline{\mathcal{C}}_{i}, \overline{\mathcal{S}}_{i}$ of $\bar{X}$ ). Finally, as $L\left(P_{i}\right)=\left\{\xi_{i}\right\}$, for every $1 \leq i \leq m$ we can find an element $\gamma_{i} \in \Gamma$, with $\ell_{i}=d\left(x, \gamma_{i} x\right)$, which is in Schottky position with $P_{i}$ relatively to $x$, i.e. such that the domains of attraction $\mathcal{U}^{ \pm}\left(\gamma_{i}\right)=\left\{y \mid d\left(\gamma_{i}^{ \pm 1} x, y\right) \leq d(x, y)\right\}$ are included in the Dirichlet domain $\mathcal{D}\left(P_{i}, x\right)$, as in Proposition 2.10.

For the following, we will then set $d=\max \left\{\operatorname{diam}(\mathcal{K}), \operatorname{diam}\left(\mathcal{S}_{i}\right), 1 / \operatorname{depth}\left(H_{\xi_{i}}\right), \ell_{i}\right\} \geq \epsilon_{0}$. 


\section{Proposition 3.1 (Counting Formula)}

Assume that $x, y \in X$ project respectively to the compact core $\overline{\mathcal{K}}$ and to a cuspidal end $\overline{\mathcal{C}}_{i}$ of $\bar{X}=\Gamma \backslash X$. There exists $C^{\prime \prime}=C(n, a, b, d)$ such that:

$$
\left[v_{\Gamma}(x, \cdot) * v_{P_{i}}(x, y, \cdot)\right]\left(R-D_{0}\right) \stackrel{C^{\prime \prime}}{\prec} v_{\Gamma}(x, y, R) \stackrel{C^{\prime \prime}}{\prec}\left[v_{\Gamma}(x, \cdot) * v_{P_{i}}(x, y, \cdot)\right]\left(R+D_{0}\right) \quad \forall R \geq 0
$$

for a constant $D_{0}$ only depending on $n, a, b, d$.

Proof. We will write, as usual, $|\gamma|_{x}=d(x, \gamma x)$ and $|\gamma P|_{x}=d(x, \gamma P x)$, and choose a constant $\Delta>\max \left\{R_{0}, \Delta_{0}, 2 \delta_{0}+4 d\right\}$, where $R_{0}, \Delta_{0}, \delta_{0}$ are the constants of Propositions 2.5 and 2.10. We first show that

$$
B(x, R) \cap \Gamma y \subset \bigcup_{k=1}^{N} \bigcup_{\bar{\gamma} \in \Gamma,|\bar{\gamma}| \leq k \Delta} B(\bar{\gamma} x,(N-k) \Delta) \cap\left(\bar{\gamma} P_{i}\right) y
$$

for $N=\left\lfloor\frac{R}{\Delta}\right\rfloor+2$. Actually, let $\gamma y \in B(x, R) \cap \gamma H_{\xi_{i}}$ and set $\bar{y}_{i}=[x, \gamma \xi] \cap \gamma \partial H_{\xi_{i}}$. By using the action of the group $\gamma P_{i} \gamma^{-1}$ on $\gamma H_{\xi_{i}}$, we can find $\bar{\gamma}=\gamma p$, with $p \in P_{i}$, such that $\bar{y}_{i} \in \bar{\gamma} \mathcal{C}_{i}$. Since the angle $\angle \bar{y}_{i}(x, \gamma y)$ at $\bar{y}_{i}$ is greater than $\frac{\pi}{2}$, we have:

$$
d(x, \gamma y) \leq d\left(x, \bar{y}_{i}\right)+d\left(\bar{y}_{i}, \gamma y\right) \leq d(x, \gamma y)+\epsilon_{0}<R+\epsilon_{0}
$$

with $|\bar{\gamma}| \leq d\left(x, \bar{y}_{i}\right)+d<R+d+\epsilon_{0} \leq N \Delta$. Then, if $k \Delta \leq|\bar{\gamma}|<(k+1) \Delta$, we deduce

$$
d(\bar{\gamma} x, \gamma y) \leq d\left(\bar{y}_{i}, \gamma y\right)+d \leq R+\epsilon_{0}-d(x, \bar{\gamma} x)+2 d<(N-k) \Delta
$$

which shows that $\gamma y=\bar{\gamma} p^{-1} y \in B(\bar{\gamma} x,(N-k) \Delta) \cap\left(\bar{\gamma} P_{i}\right) y=\bar{\gamma}\left[B(x,(N-k) \Delta) \cap P_{i} y\right]$. Thus, we obtain:

$$
v_{\Gamma}(x, y, R) \leq \sum_{k=1}^{N} v_{\Gamma}(x, k \Delta) \cdot v_{P_{i}}(x, y,(N-k) \Delta) \prec v_{\Gamma} * v_{P_{i}}(R+2 \Delta)
$$

This proves the right hand side of our inequality.

The left hand is more delicate, as we need to dissect the ball $B(x, R)$ in disjoint annuli. So, consider the set $\widehat{\Gamma}_{i}$ of minimal representatives of $\Gamma / P_{i}$ as in the proof of Proposition 2.10. We have:

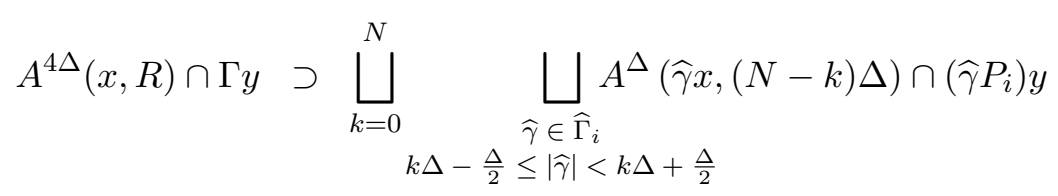

for $N=\left\lfloor\frac{R}{\Delta}\right\rfloor+1$. In fact, given $\gamma y=\widehat{\gamma} p_{i} y \in A^{\Delta}(\widehat{\gamma} x,(N-k) \Delta)$ with $\widehat{\gamma} x \in A^{\Delta}(x, k \Delta)$ we have again

$$
N \Delta-2 \Delta \leq|\widehat{\gamma}|+d(\widehat{\gamma} x, \gamma y)-2 d-\epsilon_{0} \leq d(x, \gamma y) \leq|\widehat{\gamma}|+d(\widehat{\gamma} x, \gamma y)<N \Delta+\Delta
$$

as $\Delta>2 d+\epsilon_{0}$, hence $\gamma y \in A^{4 \Delta}(x, R)$. Notice that (11) is a disjoint union, as the annuli with the same center do not intersect by definition, while for $\widehat{\gamma} \neq \widehat{\gamma}^{\prime}$ the orbits 
$\widehat{\gamma} P_{i} y$ and $\widehat{\gamma}^{\prime} P_{i} y$ lie on different horospheres $\widehat{\gamma} H_{i} \neq \widehat{\gamma}^{\prime} H_{i}$, which are disjoint by Margulis' Lemma. From (11) and by Proposition 2.10 we deduce that for all $R>0$ it holds:

$$
v_{\Gamma}^{4 \Delta}(x, y, R) \geq \frac{1}{2} \sum_{k=0}^{N} v_{\Gamma}^{\Delta / 2}(x, k \Delta) \cdot v_{P_{i}}^{\Delta}(x, y,(N-k) \Delta)
$$

as $\Delta>2 \ell_{i}$. Now, we set $h_{i}=b_{\xi_{i}}(x, y)$ and we sum (12) over annuli of radii $R_{n}=n \Delta$, and we get:

$$
\begin{aligned}
v_{\Gamma}(x, y, R) & \geq \frac{1}{4} \sum_{n=0}^{\left\lfloor\frac{R}{\Delta}\right\rfloor-2} v_{\Gamma}^{4 \Delta}(x, y, n \Delta) \succ \sum_{k=0}^{\left\lfloor\frac{R}{\Delta}\right\rfloor-1}\left[\sum_{n \geq k}^{\left\lfloor\frac{R}{\Delta}\right\rfloor-1} v_{\Gamma}^{\Delta / 2}(x,(n-k) \Delta)\right] \cdot v_{P_{i}}^{\Delta}(x, y, k \Delta) \geq \\
& \geq \sum_{k \geq \frac{h_{i}}{\Delta}+1}^{\left\lfloor\frac{R}{\Delta}\right\rfloor-1} v_{\Gamma}(x, R-(k+2) \Delta) \cdot v_{P_{i}}^{\Delta}(x, y, k \Delta) \stackrel{C^{\prime}}{\succ} \sum_{k=\frac{h_{i}}{\Delta}+1}^{\left\lfloor\frac{R}{\Delta}+1\right.} \frac{v_{\Gamma}(x, R-(k+2) \Delta)}{\mathcal{A}_{P_{i}}\left(x, \frac{k \Delta+h_{i}}{2}\right)}
\end{aligned}
$$

as $v_{P_{i}}^{\Delta}(x, y, k \Delta) \succ \mathcal{A}_{P_{i}}^{-1}\left(x, \frac{k \Delta+h_{i}}{2}\right)$ if $k \Delta \geq h_{i}+\Delta>h_{i}+R_{0}$ by Proposition 2.5.

Using again Proposition 2.5 and (4), it is easily verified that the expression in (13) is greater than the continuous convolution $v_{\Gamma}(x, \cdot) * v_{P_{i}}(x, y, \cdot)(R+4 \Delta)$, up to a multiplicative constant $C C^{\prime} \Delta$. This ends the proof, by taking $D_{0}=4 \Delta$. $\square$

The Counting Formula enables us to reduce the estimate of the growth function $v_{X}$ to a group-theoretical calculus, that is to the estimate of a the convolution of $v_{\Gamma}$ with the cuspidal functions $\mathcal{F}_{P_{i}}$ of maximal parabolic subgroups $P_{i}$ of $\Gamma$ :

\section{Proposition 3.2 (Volume Formula)}

There exists a constant $C^{\prime \prime \prime}=C^{\prime \prime \prime}(n, a, b, d, \operatorname{vol}(\mathcal{K}))$, such that:

$\left[v_{\Gamma}(x, \cdot) * \sum_{i} \mathcal{F}_{P_{i}}(x, \cdot)\right]\left(R-2 D_{0}\right) \stackrel{C^{\prime \prime \prime}}{\prec} v_{X}(x, R) \stackrel{C^{\prime \prime \prime}}{\prec}\left[v_{\Gamma}(x, \cdot) * \sum_{i} \mathcal{F}_{P_{i}}(x, \cdot)\right]\left(R+2 D_{0}\right) \quad \forall R \geq 0$

for $D_{0}=D_{0}(n, a, b, d)$ as in Proposition 3.1.

Proof. Let $h_{i}=d\left(x, H_{\xi_{i}}\right)$; we may assume that the constants $R_{0}, D_{0}$ of Propositions 2.5 and 3.1 satisfy $D_{0} \gg d \geq \operatorname{diam}(\mathcal{K}) \geq h_{i} \gg R_{0}$. Now call $\mathcal{S}_{i}(h)=\psi_{\xi_{i}, h}\left[\mathcal{S}_{i}\right] ;$ integrating $v_{\Gamma}(x, y, R)$ over the fundamental domain $\mathcal{D}$ yields, by Proposition 3.1:

$$
\begin{aligned}
v_{X}(x, R+2 & \left.D_{0}\right)=\int_{\mathcal{D}} v_{\Gamma}\left(x, y, R+2 D_{0}\right) d y=\int_{\mathcal{K}} v_{\Gamma}\left(x, y, R+2 D_{0}\right) d y+\sum_{i=1}^{m} \int_{\mathcal{C}_{i}} v_{\Gamma}\left(x, y, R+2 D_{0}\right) d y \\
& C^{\prime \prime} \sum_{i=1}^{m} \int_{2 h_{i}}^{R+D_{0}} v_{\Gamma}\left(x, R+2 D_{0}-t\right)\left[\int_{h_{i}}^{t-h_{i}} \int_{\mathcal{S}_{i}(h)} v_{P_{i}}(x, y, t) d y d h\right] d t
\end{aligned}
$$

which then gives by Propositions 2.5 and 2.6, as $h=b_{\xi_{i}}(x, y) \leq t-h_{i}<t-R_{0}$,

$$
\int_{2 h_{i}}^{R+D_{0}} v_{\Gamma}\left(x, R+2 D_{0}-t\right)\left[\sum_{i=1}^{m} \int_{h_{i}}^{t-h_{i}} \frac{\mathcal{A}_{P_{i}}(x, h)}{\mathcal{A}_{P_{i}}\left(x, \frac{t+h}{2}\right)} d h\right] d t
$$




$$
\geq \int_{0}^{R+D_{0}-2 h_{i}} v_{\Gamma}(x, R-t)\left[\sum_{i=1}^{m} \int_{0}^{t} \frac{\mathcal{A}_{P_{i}}\left(x, s+h_{i}\right)}{\mathcal{A}_{P_{i}}\left(x, \frac{t+s+3 h_{i}}{2}\right)} d s\right] d t \succ \int_{0}^{R} v_{\Gamma}(x, R-t) \sum_{i=1}^{m} \mathcal{F}_{P_{i}}(x, t) d t .
$$

Reciprocally, we have $v_{\Gamma}\left(x, R-D_{0}\right) \leq v_{\Gamma}(x, y, R) \leq v_{\Gamma}\left(x, R+D_{0}\right)$ so again by Proposition 3.1 and Remarks 2.7 we obtain

$$
\begin{aligned}
v_{X}\left(x, R-2 D_{0}\right) & \stackrel{C^{\prime \prime}}{\prec} \operatorname{vol}(\mathcal{K}) \cdot v_{\Gamma}\left(x, R-D_{0}\right)+\sum_{i=1}^{m} \int_{\mathcal{C}_{i}}\left[\int_{0}^{R-2 D_{0}} v_{\Gamma}(x, t) v_{P_{i}}(x, y, R-t) d t\right] d y \\
& C^{\prime \prime \prime \prime} v_{\Gamma}\left(x, R-D_{0}\right)+\int_{0}^{R-2 D_{0}} v_{\Gamma}(x, t)\left[\sum_{i=1}^{m} \int_{0}^{R-t} \frac{\mathcal{A}_{P_{i}}(x, h)}{\mathcal{A}_{P_{i}}\left(x, \frac{R-t+h}{2}\right)} d h\right] d t
\end{aligned}
$$

as $v_{P_{i}}(x, y, R-t)=0$ for $R-t<b_{\xi_{i}}(x, y)=h$. This proves the converse inequality, since $v_{\Gamma}\left(x, R-D_{0}\right) \prec v_{\Gamma}\left(x, R-D_{0}\right) \mathcal{F}_{P_{i}}\left(R_{0}\right) \leq \frac{1}{D_{0}-R_{0}} \int_{R-D_{0}}^{R-R_{0}} v_{\Gamma}(x, t) \mathcal{F}_{P_{i}}(x, R-t) d t$.

As a consequence of the Volume Formula and of Corollary 2.8, we deduce ${ }^{5}$ :

Corollary 3.3 If $\mathcal{F}_{P_{i}}$ are the cuspidal functions of the parabolic subgroups of $\Gamma$ :

(i) $\omega^{+}(X)=\max \left\{\delta(\Gamma), \omega^{+}\left(\mathcal{F}_{P_{1}}\right), \ldots, \omega^{+}\left(\mathcal{F}_{P_{m}}\right)\right\}$.

(ii) $\omega^{+}(X)=\omega^{-}(X)=\delta(\Gamma)$ if $\Gamma$ is $\frac{1}{2}$-parabolically pinched.

\section{Margulis function for regular lattices}

In this section we assume that $\Gamma$ is a lattice which is neither sparse nor exotic.

To prove the the divergence of the Poincaré series of $\Gamma$, we will need a general criterion which can be found in [12], [15]:

Divergence Criterion. Let $\Gamma$ be a geometrically finite group: if $\delta^{+}(P)<\delta(\Gamma)$ for every parabolic subgroup $P$ of $\Gamma$, then $\Gamma$ is divergent.

From the divergence, we will then deduce the finiteness of the Bowen-Margulis measure by the following result, due to Dal'Bo-Otal-Peigné (see [12]):

Finiteness CRIterion. Let $\Gamma$ be a divergent, geometrically finite group, $\bar{X}=\Gamma \backslash X$. We have $\mu_{B M}(U \bar{X})<\infty$ if and only if for every maximal parabolic subgroup $P$ of $\Gamma$

$$
\sum_{p \in P} d(x, p x) e^{-\delta(\Gamma) d(x, p x)}<+\infty .
$$

\footnotetext{
${ }^{5}$ Part (i) of this corollary already appears in [14], where an upper estimate for $v_{X}$ is proved. Notice that in [14] we erroneously stated that also $\omega^{-}(X)=\max \left\{\delta(\Gamma), \omega^{-}\left(\mathcal{F}_{P_{1}}\right), \ldots, \omega^{-}\left(\mathcal{F}_{P_{m}}\right)\right\}$; an explicit counterexample to this is given in Example 5.2.
} 
Proof of Theorem 1.2. Let $\Gamma$ be a nonuniform lattice of $X$ which is neither sparse nor exotic. As $\Gamma$ is not exotic, it satisfies the gap property $\delta(P)<\delta(\Gamma)$ for all parabolic subgroups; by the Divergence and Finiteness Criterion recalled in $\S 1$, we deduce that the group is divergent and that $\mu_{B M}(U \bar{X})<\infty$. Therefore $v_{\Gamma}(x, R) \stackrel{c_{\Gamma}(x)}{\asymp}$ $e^{\delta(\Gamma) R}$ is purely exponential (for some $c_{\Gamma}(x)$ depending on $\Gamma, x$ ). We will now show that $X$ has a Margulis function.

Let $\mathcal{D}$ be the fundamental domain for $\Gamma$ and $P_{i}$ the maximal parabolic subgroup fixing $\xi_{i}$ as at the beginning of $\S 3$ : we call $w(x, y, R)=v_{\Gamma}(x, y, R) e^{-\delta(\Gamma) R}$, so that have

$$
\frac{v_{X}(x, R)}{e^{\delta(\Gamma) R}}=\int_{\mathcal{D}} \frac{v_{\Gamma}(x, y, R)}{e^{\delta(\Gamma) R}} d y=\int_{\mathcal{K}} w(x, y, R) d y+\sum_{i=1}^{m} \int_{\mathcal{C}_{i}} w(x, y, R) d y
$$

We know that $v_{\Gamma}(x, y, R) \leq v_{\Gamma}(x, R+d) \leq c_{\Gamma}(x) e^{\delta(\Gamma) R}$ for $y \in \mathcal{K}$, so we can pass to the limit for $R \rightarrow \infty$ under the integral sign in the first term. For the integrals over the cusps, we have:

$$
w(x, y, R) \stackrel{C^{\prime \prime}}{\prec} \frac{\left[v_{\Gamma}(x, \cdot) * v_{P_{i}}(x, y, \cdot)\right]\left(R+D_{0}\right)}{e^{\delta(\Gamma) R}} \stackrel{c_{\Gamma}(x)}{\prec} \int_{b_{\xi_{i}}(x, y)}^{\infty} \frac{e^{-\delta(\Gamma) t}}{\mathcal{A}_{P_{i}}\left(x, \frac{b_{\xi_{i}}(x, y)+t}{2}\right)} d t=w(x, y)
$$

Notice that the dominating function $w(x, y)$ is finite as $\delta^{+}\left(P_{i}\right)<\delta(\Gamma)$.

We will now show that $w(x, y) \in L^{1}\left(\mathcal{C}_{i}\right)$. With the same notations $h_{i}=d\left(x, H_{\xi_{i}}\right)$ and $\mathcal{S}_{i}(h)=\psi_{\xi_{i}, h}\left(\mathcal{S}_{i}\right)$ as before, we have for all $i$ :

$$
\begin{aligned}
\int_{\mathcal{C}_{i}} w(x, y) d y & =\int_{h_{i}}^{\infty} \int_{y \in \mathcal{S}_{i}(h)}\left[\int_{b_{\xi_{i}}(x, y)}^{\infty} \frac{e^{-\delta(\Gamma) t}}{\mathcal{A}_{P_{i}}\left(x, \frac{b_{\xi_{i}}(x, y)+t}{2}\right)} d t\right] d y d h=\int_{h_{i}}^{\infty} \int_{h}^{\infty} \frac{e^{-\delta(\Gamma) t} \mathcal{A}_{P_{i}}(h)}{\mathcal{A}_{P_{i}}\left(x, \frac{h+t}{2}\right)} d t d h \\
& =\int_{h_{i}}^{\infty} e^{-\delta(\Gamma) t}\left[\int_{h_{i}}^{t} \frac{\mathcal{A}_{P_{i}}(h)}{\mathcal{A}_{P_{i}}\left(x, \frac{h+t}{2}\right)} d h\right] d t \stackrel{C}{\prec} \int_{h_{i}}^{\infty} e^{-\delta(\Gamma) t} \mathcal{F}_{P_{i}}(t) d t
\end{aligned}
$$

which converges, as $\Gamma$ is not sparse and so $\omega^{+}\left(\mathcal{F}_{P_{i}}\right) \leq \delta^{+}\left(P_{i}\right)<\delta(\Gamma)$, by Corollary 2.8. We therefore obtain from (16), by dominated convergence, using Roblin's asymptotics

$$
\lim _{R \rightarrow+\infty} \frac{v_{X}(x, R)}{e^{\delta(\Gamma) R}}=\frac{\left\|\mu_{x}\right\|}{\delta(\Gamma)\left\|\mu_{B M}\right\|} \int_{\mathcal{D}}\left\|\mu_{y}\right\| d y=: m(x)<+\infty .
$$

Notice that $m(x)$ defines an $L^{1}$-function on $\bar{X}=\Gamma \backslash X$, as its integral over $\mathcal{D}$ is finite. $\square$

Proof of Theorem 1.3(i). We assume now that $X$ has an exotic lattice $\Gamma$, with the dominant parabolic subgroups $P_{i}$, for $i=1, \ldots, d$, satisfying $\delta:=\delta(\Gamma)=\delta^{+}\left(P_{i}\right) \leq$ $2\left(\delta^{-}\left(P_{i}\right)-\epsilon\right)$, for some $\epsilon>0$. By Theorem 1.2 in [14], it holds $\omega(X)=\delta(\Gamma)=\delta$.

When $\mu_{B M}(U \bar{X})<\infty$, the same lines of the above proof apply: $v_{\Gamma}(x, R) \asymp$ $c_{\Gamma}(x) e^{\delta R}$ is purely exponential, and for the same functions $w(x, y, R), w(x, y)$ we again obtain (17); but we need some more work to deduce that, for the dominant cusps $P_{i}$, the integral of $e^{-\delta t} \mathcal{F}_{P_{i}}(t)$ converges. So, for every dominant subgroup $P_{i}$, we write $v_{P_{i}}(x, t)=o_{i}(t) e^{\delta t}$, for some subexponential functions $o_{i}(t) ;$ so, $\mathcal{A}_{P_{i}}(x, t) \asymp e^{-2 \delta t} / o_{i}(2 t)$ for $t \geq R_{0}$. As $\Gamma$ is exotic, the dominant parabolic subgroups $P_{i}$ are convergent: 
actually, for any divergent subgroup $\Gamma_{0}<\Gamma$ with limit set $L\left(\Gamma_{0}\right) \subsetneq L(\Gamma)$ one has $\delta\left(\Gamma_{0}\right)<\delta(\Gamma)$ (see [13]). Therefore, the Poincaré series of $P_{i}$ gives, for $\Delta>\Delta_{0} \gg 0$

$$
\infty>\sum_{p \in P_{i}} e^{-\delta d(x, p x)} \succ \sum_{k \geq 1} \frac{v_{P_{i}}^{\Delta}(x, k \Delta)}{e^{\delta k}} \asymp \int_{\Delta}^{\infty} o_{i}(t) d t
$$

by Proposition 2.5, so the functions $o_{i}(t)$ are integrable. This shows that

$$
w(x, y)=\int_{b_{\xi_{i}}(x, y)}^{\infty} \frac{e^{-\delta t}}{\mathcal{A}_{P_{i}}\left(x, \frac{b_{\xi_{i}}(x, y)+t}{2}\right)} d t=e^{\delta b_{\xi_{i}}(x, y)} \int_{b_{\xi_{i}}(x, y)}^{\infty} o_{i}(h+t) d t<\infty
$$

Moreover, as every dominant $P_{i}$ is strictly $\frac{1}{2}$-pinched, we have $v_{P_{i}}(x, t) \succ e^{\frac{1}{2}(\delta+\epsilon) t}$ for some $\epsilon>0$, that is $\mathcal{A}_{P_{i}}(x, t) \prec e^{-(\delta+\epsilon) t}$ for all $t>0$. Then Proposition 2.6 yields

$$
\mathcal{F}_{P_{i}}(R) \asymp \int_{0}^{R} \frac{\mathcal{A}_{P_{i}}(s)}{\mathcal{A}_{P_{i}}\left(\frac{s+R}{2}\right)} d s \prec e^{\delta R} \int_{0}^{R} e^{-\epsilon s} o_{i}(s+R) d s \quad \text { for } R \gg 0
$$

hence (17) gives in this case:

$\int_{\mathcal{C}_{i}} w(x, y) d y \stackrel{C}{\prec} \int_{h_{i}}^{\infty} e^{-\delta(\Gamma) t} \mathcal{F}_{P_{i}}(t) d t \asymp \int_{h_{i}}^{\infty}\left[\int_{0}^{t} e^{-\epsilon s} o_{i}(s+t) d s\right] d t \leq \int_{0}^{\infty} e^{-\epsilon s}\left[\int_{s}^{\infty} o_{i}(s+t) d t\right] d s$ which converges, since $o_{i}$ is integrable. We can therefore pass to the limit for $R \rightarrow \infty$ under the integral in (16), obtaining the asymptotics for $v_{X}(x, R)$ as before.

On the other hand, if $\mu_{B M}(U \bar{X})=\infty$, then $v_{\Gamma}(x, R)=o_{\Gamma}(R) e^{\delta R}$ is lower-exponential, and by (18) we have $\mathcal{F}_{P_{i}}(x, R)=f_{i}(R) e^{\delta R}$ with $f_{i}(R)=\int_{0}^{R} e^{-\epsilon s} o_{i}(s+R) d s$ for the dominant cusps, and $f_{i}(R) \prec e^{-\epsilon R}$, with $\epsilon>0$, for the others; in both cases, $f_{i} \in L^{1}$, since the functions $o_{i}(t)$ are subexponential. Proposition 3.2 then gives, for any arbitrarily small $\varepsilon^{\prime}>0$

$$
\begin{aligned}
\frac{v_{X}(x, R)}{e^{\delta R}} & \prec \frac{1}{e^{\delta R}} \int_{0}^{R} v_{\Gamma}(x, t) \sum_{i} \mathcal{F}_{P_{i}}(R-t) d t \prec \int_{0}^{R} o_{\Gamma}(t) \sum_{i} f_{i}(R-t) d t \\
\leq & \sum_{i}\left\|f_{i}\right\|_{1} \cdot \sup _{t>\frac{R}{2}} o_{\Gamma}(t)+\left\|o_{\Gamma}\right\|_{\infty} \cdot \sum_{i} \int_{R / 2}^{R} f_{i}(t) d t \leq \varepsilon^{\prime} \cdot\left(\sum_{i}\left\|f_{i}\right\|_{1}+\left\|o_{\Gamma}\right\|_{\infty}\right)
\end{aligned}
$$

provided that $R \gg 0$, since $o_{\Gamma}(t)$ is infinitesimal and the $f_{i}$ are integrable. This shows that $v_{X}(x, R)$ is lower-exponential too. $\square$

REMARK 4.1 We have seen that, if $\mu_{B M}(U \bar{X})=\infty$, then $v_{\Gamma}(x, R)=o_{\Gamma}(R) e^{\delta R}$ and $v_{X}(x, R)=o_{X}(R) e^{\delta R}$, where $o_{\Gamma}, o_{X}$ are infinitesimal, and that $\mathcal{F}_{P_{i}}(x, R)=f_{i}(R) e^{\delta R}$ with $f_{i} \in L^{1}$; so,

$$
\left\|o_{\Gamma}\right\|_{1} \prec\left\|o_{X}\right\|_{1} \leq \int_{0}^{\infty} \frac{v_{X}(x, R)}{e^{\delta R}} d R \prec \int_{0}^{\infty} \int_{0}^{R} o_{\Gamma}(t) \sum_{i} f_{i}(R-t) d t d R \leq\left\|o_{\Gamma}\right\|_{1} \cdot \sum_{i}\left\|f_{i}\right\|_{1}
$$

and we can say that $o_{\Gamma}$ is $L^{1}$ if and only if $o_{X}$ is. 
Finally, in order to prove Theorem 1.1, we need to recall a characterization of constant curvature spaces as those pinched, negatively curved spaces whose lattices realize the least possible value for the entropy. The minimal entropy problem has a long history and has been declined in many different ways so far; see [23], [4], [10] for the analogue of the following statement in the compact case, and [17] for a proof in the finite-volume case:

Theorem 4.2 Let $\Gamma$ be a lattice in a Hadamard manifold $X$ with pinched curvature $-b^{2} \leq K_{X} \leq-a^{2}<0$. Then $\delta(\Gamma) \geq(n-1) a$, and $\delta(\Gamma)=(n-1) a$ if and only if $X$ has constant curvature $-a^{2}$.

Proof of Theorem 1.1. Assume that $\Gamma$ is a nonuniform lattice in a $\frac{1}{4}$-pinched negatively curved manifold $X$, i.e. $-b^{2} \leq K_{X} \leq-a^{2}$ with $b^{2} \leq 4 a^{2}$. If $X=\mathbb{H}_{a}^{n}$, then clearly $v_{X}(x, R) \asymp v_{\Gamma}(x, R)$ is purely exponential, $X$ has a Margulis function, and $\Gamma$ is divergent. Otherwise, let $P_{i}$ be the maximal parabolic subgroups of $\Gamma$, up to conjugacy. By the formulas (4), we know that for all $x \in X e^{-(n-1) b R} \prec \mathcal{A}_{P_{i}}(x, R) \prec e^{-(n-1) a R}$, so by Proposition 2.5 we have

$$
\frac{a(n-1)}{2} \leq \delta^{-}\left(P_{i}\right) \leq \delta^{+}\left(P_{i}\right) \leq \frac{b(n-1)}{2}
$$

for all $P_{i}$. Thus, $\Gamma$ is parabolically $\frac{1}{2}$-pinched. It follows from Corollary 3.3 that $\omega^{+}(X)=\omega^{-}(X)=\delta(\Gamma)$. Moreover, for all $P_{i}$ we have

$$
\delta^{+}\left(P_{i}\right) \leq \frac{b(n-1)}{2} \leq a(n-1)<\omega(X)=\delta(\Gamma)
$$

where the strict inequality follows by the rigidity Theorem 4.2, since $X \neq \mathbb{H}_{a}^{n}$. The same argument applies when $\bar{X}$ is only asymptotically $\frac{1}{4}$-pinched, by replacing $-a^{2},-b^{2}$ with the bounds $-k_{+}^{2}-\epsilon \leq K_{X} \leq-k_{-}^{2}+\epsilon$ on the cusps $\overline{\mathcal{C}}_{i}$. Then, $\Gamma$ is also non-exotic, and we can conclude by Theorem 1.2 that $\Gamma$ is divergent, with finite Bowen-Margulis measure, $v_{X} \asymp v_{\Gamma}$ and $X$ has a $L^{1}$ Margulis function $m(x)$.

\section{Examples}

In this section we show that all the cases presented in Theorem 1.3 do occur, by providing examples of spaces $X$ with exotic or sparse lattices $\Gamma$ which do not admit a Margulis function, and with functions $v_{\Gamma}, v_{X}$ having different behaviour.

If $\overline{\mathcal{C}}=P \backslash H_{\xi}(o)$ is a cusp of $\bar{X}=\Gamma \backslash X$, we write the metric of $X$ in horospherical coordinates on $H_{\xi}(o) \cong \partial H_{\xi}(o) \times \mathbb{R}^{+}$as $g=T(x, t)^{2} d x^{2}+d t^{2}$, for $x \in \partial H_{\xi}(o)$ and $t=b_{\xi}(o, \cdot)$. We call the function $T(x, t)$ the analytic profile of the cusp $\overline{\mathcal{C}}$. The horospherical area $\mathcal{A}_{P}(x, t)$ is then obtained by integrating $T^{n-1}(x, t)$ over a compact fundamental domain $\mathcal{S}$ for the action of $P$ on $\partial H_{\xi}(o)$; thus, we have

$$
\mathcal{A}_{P}(x, t) \stackrel{c}{\leftrightharpoons} T^{n-1}(x, t) \quad \text { for all } x \in \overline{\mathcal{C}}
$$

(for a constant $c$ depending on $X$ and $o$ ). Also, notice that, in the particular case where $T(y, t)=T(t)$, for points $x, y$ belonging to a same horosphere $H_{\xi}$ we have by the Approximation Lemma 2.2 


$$
d(x, y) \sim 2 T^{-1}\left(\frac{T(0)}{d_{\xi}(x, y)}\right) \quad \text { for } R=d(x, y) \rightarrow \infty .
$$

We will repeatedly make use of the following lemma, which is a easy modification of one proved in [14]:

Lemma 5.1 Let $b>a>0, \beta>\alpha>0$ and $\epsilon>0$ be given.

There exist $D=D(a, b, \alpha, \beta, \epsilon)>1$ and $D^{\prime}=D^{\prime}(a, b, \alpha, \beta)>0$ such that if $[p, q],[r, s]$ are disjoint intervals satisfying $r \geq D q$ and $p \geq D^{\prime}$, then there exist $C^{2}$, convex and decreasing functions $\phi_{\epsilon}, \psi_{\epsilon}$ on $[p, s]$ satisfying:

$$
\left\{\begin{array} { l } 
{ \forall t \in [ p , q ] , \quad \phi _ { \epsilon } ( t ) = t ^ { \beta } e ^ { - b t } } \\
{ \forall t \in [ r , s ] , \quad \phi _ { \epsilon } ( t ) = t ^ { \alpha } e ^ { - a t } } \\
{ \forall t \in [ p , s ] , \quad t ^ { \beta } e ^ { - b t } \leq \phi _ { \epsilon } ( t ) \leq t ^ { \alpha } e ^ { - a t } \quad \text { and } } \\
{ \forall t \in [ p , s ] , \quad a ^ { 2 } - \epsilon \leq \frac { \phi _ { \epsilon } ^ { \prime \prime } ( t ) } { \phi _ { \epsilon } ( t ) } \leq b ^ { 2 } + \epsilon }
\end{array} \quad \left\{\begin{array}{ll}
\forall t \in[p, q], & \psi_{\epsilon}(t)=t^{\alpha} e^{-a t} \\
\forall t \in[r, s], & \psi_{\epsilon}(t)=t^{\beta} e^{-b t} \\
\forall t \in[p, s], & t^{\beta} e^{-b t} \leq \psi_{\epsilon}(t) \leq t^{\alpha} e^{-a t} \\
\forall t \in[p, s], & a^{2}-\epsilon \leq \frac{\psi_{\epsilon}^{\prime \prime}(t)}{\psi_{\epsilon}(t)} \leq b^{2}+\epsilon
\end{array}\right.\right.
$$

\section{Example 5.2 Sparse lattices.}

Sparse lattices satisfying $\omega^{+}(X)>\delta(\Gamma)$ were constructed by the authors in [14]. Here, we modify that construction to show that, for spaces $X$ admitting sparse lattices, one can have $\omega^{+}(X)>\omega^{-}(X)>\delta(\Gamma)$ (in contrast, notice that $\delta(\Gamma)$ always is a true limit); this shows in particular that sparse lattices generally do not have a Margulis function. We start from a hyperbolic surface $\bar{X}_{0}=X_{0} \backslash \Gamma$ of finite volume, homeomorphic to a 3-punctured sphere, and, for any arbitrary small $\epsilon>0$, we perturb the hyperbolic metric $g_{0}$ on one cusp $\overline{\mathcal{C}}=P \backslash H_{\xi}(x)$ into a metric $g_{\epsilon}$ by choosing an analytic profile $T_{\epsilon}$ obscillating, on infinitely many horospherical bands, from $e^{-t}$ to $e^{-b t}$.

Namely, choose $a=1, b>2$ and $\epsilon>0$ arbitrarily small, and let $D, D^{\prime}$ be the constants given by Lemma 5.1. For $M \gg 1$, we define a sequence of disjoint subintervals of $\left[M^{4 n}, M^{4 n+1}\right]$ :

$$
\left[p_{n}, q_{n}\right]:=\left[M^{4 n}, 2 M^{4 n}\right], \quad\left[r_{n}, s_{n}\right]:=\left[\frac{p_{n}+M^{4 n+1}}{2}, \frac{q_{n}+M^{4 n+1}}{2}\right]
$$

such that $r_{n} \geq D q_{n}, p_{n+1} \geq D s_{n}, p_{1} \geq D^{\prime}$ (we can choose any $M \geq \max \{4 D-1, \sqrt[3]{D}\}$ in order that these conditions are satisfied). Notice that $\frac{t+M^{4 n+1}}{2} \in\left[r_{n}, s_{n}\right]$ for all $t \in\left[p_{n}, q_{n}\right]$. Then, by Lemma 5.1 , we consider a $C^{2}$, decreasing function $T_{\epsilon}(t)$ satisfying:

(i) $T_{\epsilon}(t)=e^{-t}$ for $t \in\left[M^{4 n-2}, M^{4 n}\right] \cup\left[p_{n}, q_{n}\right]$, and $T_{\epsilon}(t)=e^{-b t}$ for $t \in\left[r_{n}, s_{n}\right]$;

(ii) $e^{-b t} \leq T_{\epsilon}(t) \leq e^{-t}$ and $-b^{2}-\epsilon \leq T_{\epsilon}^{\prime \prime}(t) / T_{\epsilon}(t) \leq-1+\epsilon$.

Thus, the new analytic profile $T_{\epsilon}(t)$ of $\overline{\mathcal{C}}$ coincides with the profile of a usual hyperbolic cusp on $\left[M^{4 n-2}, 2 M^{4 n}\right]$, and with the profile of a cusp in curvature $-b^{2}$ on the bands $\left[r_{n}, s_{n}\right] \subset\left[M^{4 n}, M^{4 n+1}\right]$. We have, with respect to the metric $g_{\epsilon}$ :

(a) $\delta^{+}(P)=\frac{b}{2}$ and $\delta^{-}(P)=\frac{1}{2}$, by (i) and (ii), because of Proposition 2.5;

(b) $\omega^{+}\left(\mathcal{F}_{P}\right) \geq \frac{b}{2}+\delta$ for $\delta=\frac{1}{M}\left(\frac{b}{2}-1\right)>0$, because for $R=M^{4 n+1}$ 
$\mathcal{F}_{P}(x, R) \succ \int_{0}^{R} \frac{\mathcal{A}_{\epsilon}(x, t)}{\mathcal{A}_{\epsilon}\left(x, \frac{t+R}{2}\right)} d t \geq \int_{p_{n}}^{q_{n}} \frac{e^{-t}}{e^{-b\left(\frac{t+R}{2}\right)}} d t \succ e^{\frac{b}{2} R} \cdot M^{4 n} e^{\left(\frac{b}{2}-1\right) p_{n}} \geq e^{\frac{b}{2} R} \cdot e^{\frac{1}{M}\left(\frac{b}{2}-1\right) R}$

as $p_{n} / R=\frac{1}{M}$;

(c) $\omega^{-}\left(\mathcal{F}_{P}\right) \leq \frac{1}{2}$ if $M>2$, as for $R \in\left[M^{4 n+3}, M^{4 n+4}\right]$ we obtain:

$$
\mathcal{F}_{P}(x, R) \prec \int_{0}^{R} \frac{e^{-t}}{e^{-\left(\frac{t+R}{2}\right)}} d t \prec e^{\frac{R}{2}}
$$

since $M^{4 n+4} \geq \frac{t+R}{2} \geq \frac{M^{4 n+3}}{2} \geq M^{4 n+2}$

(d) $\delta(\Gamma)$ is arbitrarily close to $\delta^{+}(P)$, let's say $\delta(\Gamma) \leq \frac{b}{2}+\frac{\delta}{2}$, if we perturb the hyperbolic metric sufficiently far in the cusp $\overline{\mathcal{C}}$, i.e. if $r_{1} \gg 0$ (this is Proposition 5.1 in [14]).

It follows that $\omega^{-}(X)>\delta(\Gamma)$. Actually, assume that $v_{\Gamma}(x, R) \succ e^{(\delta(\Gamma)-\eta) R}$, for arbitrarily small $\eta$. By Proposition 3.2 and (20), we deduce that for any $R \gg 0$, if $M^{4 n+1} \leq R<M^{4 n+5}$

$$
v_{X}\left(x, R+2 D_{0}\right) \geq v_{\Gamma}(x, \cdot) * \mathcal{F}_{P}(x, \cdot)(x, R) \succ e^{(\delta(\Gamma)-\eta)\left(R-M^{4 n+1}\right)} \cdot e^{\left(\frac{b}{2}+\delta\right) M^{4 n+1}}
$$

by taking just the term $\left.v_{\Gamma}(x, R-t) \mathcal{F}_{P}(x, t)\right)$ of the convolution with $t$ closest to $M^{4 n+1}$, where $\mathcal{F}_{P}(t) \succ e^{\left(\frac{b}{2}+\delta\right) t}$; as $M^{4 n+1} \geq R / M^{4}$ we get $v_{X}(x, R+2 \Delta) \succ e^{\left(\delta(\Gamma)-\eta+\frac{\delta / 2+\eta}{M^{4}}\right) R}$ which gives $\omega^{-}(X) \geq \delta(\Gamma)+\frac{\delta}{2 M^{4}}, \eta$ being arbitrary.

Finally, we show that $\omega^{+}(X)>\omega^{-}(X)$. In fact, the cusps different from $\overline{\mathcal{C}}$ being hyperbolic, we have, always by Proposition 3.2, that $\omega^{+}(X)=\omega^{+}\left(\mathcal{F}_{P}\right) \geq \frac{b}{2}+\delta$.

On the other hand, we know that $\omega^{+}\left(\mathcal{F}_{P}\right) \leq \max \left\{\delta^{+}(P), 2\left(\delta^{+}(P)-\delta^{-}(P)\right\}=b-1\right.$, by Corollary 2.8; thus, assuming $\mathcal{F}_{P}(x, t) \prec e^{(b-1+\eta) t}$, for arbitrarily small $\eta$, equation (21) yields for $R=M_{4 n+4}$

$$
\begin{aligned}
v_{X}\left(x, R-2 D_{0}\right) & \leq \int_{0}^{M^{4 n+3}} v_{\Gamma}(x, R-t) \cdot \mathcal{F}_{P}(x, t) d t+\int_{M^{4 n+3}}^{R} v_{\Gamma}(x, R-t) \cdot \mathcal{F}_{P}(x, t) d t \\
& \prec \int_{0}^{M^{4 n+3}} e^{\delta(\Gamma)(R-t)} \cdot e^{(b-1+\eta) t} d t+\int_{M^{4 n+3}}^{R} e^{\delta(\Gamma)(R-t)} \cdot e^{\frac{1}{2} t} d t \\
& \prec e^{\delta(\Gamma) R} \cdot e^{(b-1+\eta-\delta(\Gamma)) M^{4 n+3}} \leq e^{\left(\frac{b}{2}+\frac{\delta}{2}+\frac{(b / 2+\eta-1}{M}\right) R}
\end{aligned}
$$

being $\frac{b}{2} \leq \delta(\Gamma) \leq \frac{b}{2}+\frac{\delta}{2}$ and $M^{4 n+3}=\frac{R}{M}$. Hence $\omega^{-}(X)<\frac{b}{2}+\delta \leq \omega^{+}(X)$, if $M \gg 0$ and $\eta$ small enough.

Examples 5.3 Exotic, strictly $\frac{1}{2}$-parabolically pinched lattices.

We say that a lattice $\Gamma$ is strictly $\frac{1}{2}$-parabolically pinched when every parabolic sugroup $P<\Gamma$ satisfies the strict inequality $\delta^{+}(P)<2 \delta^{-}(P)$. Let $\bar{X}=\Gamma \backslash X$ as before; we show here that, for $\Gamma$ exotic and strictly $\frac{1}{2}$-parabolically pinched, the following cases which appear in Theorem 1.3 do occur: 
(a) $\mu_{B M}(U \bar{X})=\infty$ and $v_{X}$ is lower-exponential;

(b) $\mu_{B M}(U \bar{X})<\infty$ and $v_{X}$ is purely exponential.

We start by an example of lattice satisfying (a).

In [16] the authors show how to construct convergent lattices, in pinched negative curvature and any dimension $n$; we will take $n=2$ here by the sake of simplicity. In those examples, the metric is hyperbolic everywhere but one cusp $\mathcal{C}$, which has analytic profile $T(t)=t^{\beta} e^{b t}$ for $t \geq t_{0} \gg 0$, with $\beta>1$ and $b>2$. Therefore, there is just one dominant maximal parabolic subgroup $P$, with $\mathcal{A}_{P}(x, t) \asymp T(t) \asymp e^{b t}$, and $\delta^{+}(P)=\delta^{-}(P)=\frac{b}{2}$; moreover, the subgroup $P$ is convergent as

$$
\sum_{p \in P} e^{-\frac{b}{2} d(x, p x)} \leq \sum_{k \geq 0} v_{P}(x, k) e^{-\frac{b}{2} k} \asymp \int_{1}^{\infty} \frac{e^{-\frac{b}{2} t}}{\mathcal{A}_{P}\left(x, \frac{t}{2}\right)} d t \asymp \int_{1}^{\infty} \frac{e^{-\frac{b}{2} t}}{(t)^{\beta} \cdot e^{-\frac{t}{2}}} d t \asymp \int_{1}^{\infty} t^{-\beta} d t<\infty .
$$

By decomposing the elements of $\Gamma$ in geodesic segments which, alternatively, either go very deep in the cusp or stay in the hyperbolic part of $X$, we show in [16] that $\Gamma$ is convergent too, provided that $t_{0} \gg 0$. Then, $\Gamma$ is exotic with infinite Bowen-Margulis measure, and $v_{\Gamma}(x, R)$ is lower-exponential by Roblin's asymptotics. By Theorem 1.3(i), the function $v_{X}$ is lower-exponential as well, with the same exponential growth rate.

We now give an example for (b).

This is more subtle, as we need to take a divergent, exotic lattice $\Gamma$ : the existence of such lattices is established, in dimension 2, in [16]. Again, the simplest example is homeomorphic to a three-punctured sphere, with three cusps, and hyperbolic metric outside one cusp $\overline{\mathcal{C}}$, which has analytic profile

$$
T(t)= \begin{cases}e^{-t} & \text { for } t \leq A \\ e^{-b t} & \text { for } t \in[A, A+B]+D \\ t^{3} \cdot e^{-b t} & \text { for } t \gg D+A+B\end{cases}
$$

with $b>2$ and $A, B, D \gg 0$. As before, we have one dominant and convergent maximal parabolic subgroup $P$, with $\delta^{+}(P)=\delta^{-}(P)=\frac{b}{2}$. In [16] it is proved that, according to the values of $A$ and $B$, the behaviour of the group $\Gamma$ is very different: it is convergent with critical exponent $\delta(\Gamma)=\delta^{+}(P)$, for $A \gg 0$ and $B=0$, while it is divergent with $\delta(\Gamma)>\delta^{+}(P)$ if $B \gg A$. By perturbation theory of transfer operators, it is then proved that there exists a value of $B$ for which $\Gamma$ is divergent with $\delta(\Gamma)=\delta^{+}(P)$ precisely. Thus, for this particular value of $B$, the lattice $\Gamma$ is exotic, and has finite Bowen-Margulis measure by the Finiteness Criterion, as

$$
\sum_{p \in P} d(x, p x) e^{-\delta(\Gamma) d(x, p x)} \prec \int_{1}^{\infty} \frac{t e^{-\frac{b}{2} t}}{\mathcal{A}_{P}\left(x, \frac{t}{2}\right)} d t \prec \int_{1}^{\infty} \frac{t e^{-\frac{b}{2} t}}{t^{3} \cdot e^{-b \frac{t}{2}}} d t \asymp \int_{1}^{\infty} t^{-2} d t<\infty
$$

It follows that $v_{X} \asymp v_{\Gamma}$ is purely exponential, by Theorem 1.3(i).

Examples 5.4 Exotic, exactly $\frac{1}{2}$-parabolically pinched lattices.

We say that a lattice $\Gamma$ is exactly $\frac{1}{2}$-parabolically pinched when it is $\frac{1}{2}$-parabolically pinched and has a parabolic sugroup $P<\Gamma$ satisfisfying the quality $\delta^{+}(P)=2 \delta^{-}(P)$. We show here that for an exotic and exactly $\frac{1}{2}$-parabolically pinched lattice $\Gamma$, the following cases can occur: 
(a) $\mu_{B M}(U \bar{X})<\infty$, with $v_{\Gamma}$ purely exponential and $v_{X}$ weakly upper-exponential;

(b) $\mu_{B M}(U \bar{X})=\infty$, with $v_{\Gamma}$ lower-exponential and $v_{X}$ weakly upper-exponential.

We start by (a). Consider a surface with three cusps as in the Examples 5.3, now perturbing the hyperbolic metric on the cusp $\overline{\mathcal{C}}$ to an analytic profile defined as follows. First, choose a sequence of disjoint subintervals of $\left[M^{2 n}, M^{2 n+1}\right]$

$$
\left[p_{n}, q_{n}\right]:=\left[M^{2 n}, \mu M^{2 n+1}\right], \quad\left[r_{n}, s_{n}\right]:=\left[\frac{p_{n}+M^{2 n+1} / 2}{2}, \frac{q_{n}+M^{2 n+1}}{2}\right]
$$

and then define, for $b>1$ and $0<\gamma<1$

$$
T(t)= \begin{cases}e^{-t} & \text { for } t \leq A \\ e^{-b t} & \text { for } t \in[A, A+B]+D \\ t \cdot e^{-\frac{b}{2} t} & \text { for } t \in\left[p_{n}, q_{n}\right] \\ t^{2+\gamma} \cdot e^{-b t} & \text { for } t \in\left[r_{n}, s_{n}\right]\end{cases}
$$

with $t^{2+\gamma} e^{-b t} \leq T(t) \leq t \cdot e^{-\frac{b}{2} t}$ for all $t \geq t_{0} \gg 0$ (in order that the conditions of Lemma 5.1 are satisfied, it is enough to choose any $0<\mu<\frac{1}{4 D}$ and $M>D$ ).

As before, the profile $T$ gives a divergent, exotic lattice $\Gamma$ for a suitable value of $B$ and $A \gg 0$, with dominant parabolic subgroup $P$ having $\delta^{+}(P)=\frac{b}{2}=\delta(\Gamma)$, and $\delta^{-}(P)=\frac{b}{4}$. The Bowen-Margulis measure of $\Gamma$ is finite, as (22) also holds in this case; thus, $v_{\Gamma}$ is purely exponential. Let us now show that $v_{X}$ is upper exponential: for every $R=M^{2 n+1}$ we have, by Proposition 3.2 ,

$$
\begin{gathered}
v_{X}\left(x, R+2 D_{0}\right) \succ\left[v_{X}(x, \cdot) * \mathcal{F}_{P}(x, \cdot)\right](R) \asymp \int_{0}^{R} v_{\Gamma}(x, R-t)\left[\int_{0}^{t} \frac{\mathcal{A}_{P}(x, s)}{\mathcal{A}_{P}\left(x, \frac{s+t}{2}\right)} d s\right] d t \\
=\int_{0}^{R} \mathcal{A}_{P}(x, s)\left[\int_{s}^{R} \frac{v_{\Gamma}(x, R-t)}{\mathcal{A}_{P}\left(x, \frac{s+t}{2}\right)} d t\right] d s \geq \int_{p_{n}}^{q_{n}} \mathcal{A}_{P}(x, s)\left[\int_{\frac{R}{2}}^{R} \frac{v_{\Gamma}(x, R-t)}{\mathcal{A}_{P}\left(x, \frac{s+t}{2}\right)} d t\right] d s
\end{gathered}
$$

since $q_{n}<\frac{R}{2}$. As $\frac{s+t}{2} \in\left[r_{n}, s_{n}\right]$ if $s \in\left[p_{n}, q_{n}\right]$ and $t \in\left[\frac{R}{2}, R\right]$, by the definition of $T(t) \asymp \mathcal{A}_{P}(x, t)$ on $\left[r_{n}, s_{n}\right]$, this yields

$$
v_{X}(x, R) \succ \int_{p_{n}}^{q_{n}} s e^{-\frac{b}{2} s}\left[\int_{\frac{R}{2}}^{R} \frac{e^{\frac{b}{2}(R-t)}}{e^{-b\left(\frac{s+t}{2}\right)}(s+t)^{2+\gamma}} d t\right] d s \succ e^{\frac{b}{2} R} \int_{p_{n}}^{q_{n}} \frac{R s}{(s+R)^{2+\gamma}} d s
$$

with $\int_{p_{n}}^{q_{n}} \frac{R s}{(s+R)^{2+\gamma}} d s \geq \int_{\frac{1}{M}}^{\mu} \frac{u}{(1+u)^{2+\gamma}} d u \asymp R^{1-\gamma}$, so $v_{X}$ is upper-exponential.

Producing examples for case (b) is more difficult; for this, we will need an exotic lattice $\Gamma$ whose orbital function satisfies $v_{\Gamma}(o, R) \asymp \frac{1}{R^{\gamma}} e^{\delta(\Gamma) R}$. Lattices with lowerexponential growth and infinite Bowen-Margulis measure are investigated in [16], where a refined counting result is proved, according to the behaviour of the profile functions of the cusps (the examples in [16] are, as far as we know, the only precise estimates of the orbital function for groups with infinite Bowen-Margulis measure). Here we only give the necessary analytic profiles of the cusps in order to have a function $v_{X}$ which is exponential or upper-exponential, referring to [16] for the precise estimate of $v_{\Gamma}$. 
We again start from a hyperbolic surface $\bar{X}_{0}=X_{0} \backslash \Gamma$ with three cusps as in 5.3, and perturb now the metric on two cusps. We choose $b>2$ and $1+\gamma<\beta<2+\gamma$, and define the profiles for $\overline{\mathcal{C}}_{1}$ and $\overline{\mathcal{C}}_{2}$ as

$$
T_{1}(t)=\left\{\begin{array}{ll}
e^{-t} & \text { for } t \leq A \\
e^{-b t} & \text { for } t \in[A, A+B]+D \\
t \cdot e^{-\frac{b}{2} t} & \text { for } t \in\left[p_{n}, q_{n}\right] \\
t^{\beta} \cdot e^{-b t} & \text { for } t \in\left[r_{n}, s_{n}\right]
\end{array} \quad \text { and } \quad T_{2}(t)= \begin{cases}e^{-t} & \text { for } t \leq A \\
t^{1+\gamma} e^{-b t} & \text { for } t \gg A\end{cases}\right.
$$

for the same sequence of intervals $\left[p_{n}, q_{n}\right],\left[r_{n}, s_{n}\right]$ as in (23).

If $P_{1}, P_{2}$ are the associated maximal parabolic subgroups, we have $\delta^{-}\left(P_{1}\right)=\frac{b}{4}$ and $\delta^{+}\left(P_{1}\right)=\frac{b}{2}$, while $\delta^{+}\left(P_{2}\right)=\delta^{-}\left(P_{2}\right)=\frac{b}{2}$ by construction. It is easily verified that these parabolic subgroups are convergent as $\gamma>0$. Again, pushing the perturbation far in the cusps (i.e. choosing $A \gg 0$ ) and for a suitable value of $B$, the lattice $\Gamma$ becomes exotic and divergent; it has two dominant cusps, it is exactly $\frac{1}{2}$-parabolically pinched, and has infinite Bowen-Margulis measure, because (as $\gamma<1)$

$$
\sum_{p \in P_{2}} d(x, p x) e^{-\delta(\Gamma) d(x, p x)} \prec \int_{1}^{\infty} \frac{t e^{-\frac{b}{2} t}}{t^{1+\gamma} \cdot e^{-b \frac{t}{2}}} d t \asymp \int_{1}^{\infty} t^{-\gamma} d t=\infty .
$$

Accordingly, $v_{\Gamma}$ is lower-exponential. In [16] it is proved that the least convergent dominant parabolic subgroup determines the asymptotics of $v_{\Gamma}$; in this case, the parabolic subgroup $P_{1}$ converges faster than $P_{2}$, and the chosen profile for $\overline{\mathcal{C}}_{2}$ then gives $v_{\Gamma}(o, R) \asymp \frac{1}{R^{1-\gamma}} e^{\delta(\Gamma) R}$, provided that $\gamma \in\left(\frac{1}{2}, 1\right)$, cp. [16].

Let us now estimate $v_{X}(x, R)$, for $R=M^{2 n+1}$. Writing $T_{1}(t)=\tau^{+}(t) e^{-b t}=\tau^{-}(t) e^{-\frac{b}{2} t}$ so that $\tau^{+}(t)=t^{\beta}$ on $\left[r_{n}, s_{n}\right]$ and $\tau^{-}(t)=t$ on $\left[p_{n}, q_{n}\right]$, we compute as in case (a):

$$
\begin{aligned}
v_{X}(x, R & \left.+2 D_{0}\right) \succ\left(v_{\Gamma}(x, \cdot) * \mathcal{F}_{P_{1}}(x, \cdot)\right)(R)=\int_{0}^{R} \int_{0}^{t} \frac{\mathcal{A}_{P_{1}}(x, s)}{\mathcal{A}_{P_{1}}\left(x, \frac{t+s}{2}\right)} v_{\Gamma}(x, R-t) d t d s \\
& \asymp \int_{0}^{R} \int_{0}^{t} \frac{\tau^{-}(s) \cdot e^{-\frac{b}{2} s} \cdot e^{\frac{b}{2}(R-t)}}{\tau^{+}\left(\frac{t+s}{2}\right) \cdot(R-t)^{1-\gamma} \cdot e^{-b\left(\frac{t+s}{2}\right)}} d t d s=e^{\frac{b}{2} R} \int_{0}^{R} \tau^{-}(s)\left[\int_{s}^{R} \frac{d t}{\tau^{+}\left(\frac{t+s}{2}\right)(R-t)^{1-\gamma}}\right] d s \\
& \succ e^{\frac{b}{2} R} \int_{p_{n}=\frac{R}{M}}^{q_{n}=\mu R} s\left[\int_{\frac{R}{2}}^{R} \frac{d t}{R^{\beta}(R-t)^{1-\gamma}}\right] d s \succ\left(\mu-\frac{1}{M}\right) R^{2+\gamma-\beta} e^{\frac{b}{2} R}
\end{aligned}
$$

which is upper-exponential as $\beta<2+\gamma$.

REMARK 5.5 Notice that in all these examples $b$ can be chosen arbitrarily close to $2 a=2$. Thus, by the last condition in Lemma 5.1, the analytic profiles give metrics with curvature $-4 a^{2}-\epsilon \leq K_{X} \leq-a^{2}$, for arbitrarily small $\epsilon>0$.

\section{References}

[1] Belegradek I., Kapovitch V., (2005) Pinching estimates for negatively curved manifolds with nilpotent fundamental groups, Geometric \& Functional Analysis G.A.F.A., vol. 15, 929-938. 
[2] Besson G., Courtois G., Gallot S., (1995)Entropies et rigidités des espaces localement symétriques de courbure strictement négative, Geom. Funct. Anal. 5, no. 5, 731-799.

[3] Besson G., Courtois G., Gallot S., (1999)Lemme de Schwarz réel et applications géométriques, Acta Math. 183, no. 2, 145-169.

[4] Bonk M., Kleiner, B., (2002) Rigidity for quasi-Möbius group actions, J. Differential Geom. 61, no. 1, 81106.

[5] Buser P., Karcher H., (1981) Gromov's almost flat manifolds, Astérisque 81, S.M.F.

[6] Boland J.; Connell, C., Souto, J., (2005) Volume rigidity for finite volume manifolds, Amer. J. Math. 127, no. 3, 535-550.

[7] Bourdon, M., (1995)Structure conforme au bord et flot géodśique d'un CAT(-1)-espace, Enseign. Math. (2) 41, no. 1-2, 63102.

[8] Bowditch B.H., (1995) Geometrical finiteness with variable negative curvature, Duke Math. J. vol. 77, 229-274.

[9] Corlette K., Iozzi A. (1999) Limit sets of isometry groups of exotic hyperbolic spaces, Trans. A. M. S.vol. 351, n. 4, 1507-1530.

[10] Courtois G., (2009)Critical exponents and rigidity in negative curvature, in Géométries à courbure négative ou nulle, groupes discrets et rigidités, 293-319, Sémin. Congr., 18, Soc. Math. France, Paris.

[11] Castillon P., Sambusetti A., (2014) On asymptotically harmonic manifolds of negative curvature, Math. Zeit. 277, 3-4, 1049-1072.

[12] Dal'bo F., Otal J.P. \& Peigné M. , (2000) Séries de Poincaré des groupes géométriquement finis, Israel Journal of Math.118, 109-124.

[13] F. Dal'bo \& M. Peigné, (1996) Groupes du ping-pong et géodésiques fermées en courbure -1, Ann. Inst.Fourier, 46, $\mathrm{n}^{\circ}$ 3, 755-799.

[14] Dal'bo F., Peigné M., Picaud J.C., Sambusetti A., (2009), On the growth of nonuniform lattices in pinched negatively curved manifolds, J. für die Reine und Angew. Math. 627, 31-52.

[15] Dal’bo F., Peigné M., Picaud J.C., Sambusetti A., (2010) On the growth of quotients of Kleinian groups, Ergodic Theory and Dynamical Systems 31 no.3, 835-851.

[16] Dal'bo F., Peigné M., Picaud J.C., Sambusetti A., (2017) Convergence and counting in infinite measure, Ann. Institut Fourier 67 no.2, 483-520.

[17] Peigné M., Sambusetti A., (2017) Entropy rigidity of negatively curved manifolds of finite volume, arXiv:1702.06567.

[18] Eberlein P., Geometry of nonpositively curved manifolds, Chicago Lectures in Mathematics.

[19] HamenstäDt U., (1990) Entropy-rigidity of locally symmetric spaces of negative curvature, Ann. of Math. (2) 131 , no. 1, 35-51.

[20] Hersonsky S., PAulin F., (2004) Counting orbits in coverings of negatively curved manifolds and Hausdorff dimension of cusps excursions, Ergod.Th. \& Dynam. Sys. 24, 803-824.

[21] Heintze E., Im Hof H.C., (1977) Geometry of horospheres, J. Diff. Geom. vol. 12, 481-491. 
[22] IZEKi H., (1995) Limit sets of Kleinian groups and conformally flat Riemannian manifolds, Invent. Math. 122, no. 3, 603625.

[23] KNIEPER G., (1997) Spherical means on compact Riemannian manifolds of negative curvature, Diff.Geometry G.A.F.A., vol. 7, 755-782.

[24] KNIEPER G., (1997) On the asymptotic geometry of nonpositively curved manifolds, G.A.F.A., vol. 7, 755-782.

[25] Margulis G. A., (1969) Certain applications of ergodic theory to the investigation of manifolds of negative curvature Funkcional. Anal. i Priložen. 3 no. 4, 89-90.

[26] Nguren Phan, T. TAM, On finite volume, negatively curved manifolds, arXiv:1110.4087v2.

[27] Otal J.P., Peigné M., (2004) Principe variationnel et groupes Kleiniens, Duke Math. J., 125 n.1, 15-44.

[28] Peigné M., (2013) Autour de l'exposant de Poincaré d'un groupe Kleinien, Monographie de L'Enseignement Mathématique 43.

[29] Pollicott M., Sharp R. , (1994) Orbit counting for some discrete groups acting on simply connected manifolds with negative curvature Inventiones Math, 117, 275-302.

[30] Roblin T., (2003) Ergodicité et équidistribution en courbure négative, Mém. Soc. Math. Fr. (N.S.) No. 95 .

[31] Sambusetti A.,Asymptotic properties of coverings in negative curvature, Geometry and Topology 12 (2008), no. 1, 617-637.

[32] Storm P., (2006) The minimal entropy conjecture for nonuniform rank one lattices, Geom. Funct. Anal. 16, no. 4, 959-980.

[33] Sullivan D. , (1979) The density at infinity of a discrete group of hyperbolic motions, IHES Publ. Math. 50, 171-202.

[34] YuE, C. B., (1996) The ergodic theory of discrete isometry groups of manifolds of variable curvature, Trans. Amer. Math. Soc. 348, no.12, 4965-5005. 